\title{
Combinatorial readout of unmodified H3R2 and acetylated H3K14 by the tandem PHD finger of MOZ reveals a regulatory mechanism for HOXA9 transcription
}

\author{
Yu Qiu, ${ }^{1,2}$ Lei Liu,, ${ }^{1,2}$ Chen Zhao, ${ }^{1,2}$ Chuanchun Han, ${ }^{1,2}$ Fudong Li, ${ }^{1,2}$ Jiahai Zhang, ${ }^{1,2}$ Yan Wang, ${ }^{3}$

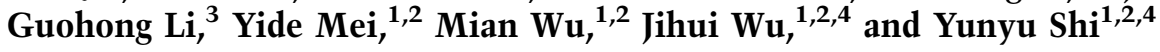 \\ ${ }^{1}$ Hefei National Laboratory for Physical Sciences at the Microscale, ${ }^{2}$ School of Life Sciences, University of Science and \\ Technology of China, Hefei, Anhui 230026, China; ${ }^{3}$ National Laboratory of Biomacromolecules, Institute of Biophysics, Chinese \\ Academy of Sciences, Beijing 100101, China
}

Histone acetylation is a hallmark for gene transcription. As a histone acetyltransferase, MOZ (monocytic leukemia zinc finger protein) is important for $\mathrm{HOX}$ gene expression as well as embryo and postnatal development. In vivo, MOZ forms a tetrameric complex with other subunits, including several chromatin-binding modules with regulatory functions. Here we report the solution structure of the tandem PHD (plant homeodomain) finger (PHD12) of human MOZ in a free state and the $1.47 \AA$ crystal structure in complex with H3K14ac peptide, which reveals the structural basis for the recognition of unmodified R2 and acetylated K14 on histone H3. Moreover, the results of chromatin immunoprecipitation (ChIP) and RT-PCR assays indicate that PHD12 facilitates the localization of MOZ onto the promoter locus of the HOXA9 gene, thereby promoting the $\mathrm{H} 3$ acetylation around the promoter region and further up-regulating the HOXA9 mRNA level. Taken together, our findings suggest that the combinatorial readout of the H3R2/K14ac by PHD12 might represent an important epigenetic regulatory mechanism that governs transcription and also provide a clue of cross-talk between the MOZ complex and histone $\mathrm{H} 3$ modifications.

[Keywords: histone acetyltransferase; MOZ; PHD finger; H3R2me0; H3K14ac; HOXA9]

Supplemental material is available for this article.

Received January 26, 2012; revised version accepted May 11, 2012.

The eukaryotic genome is assembled into chromatin. The basic unit of chromatin is the nucleosome, which is comprised of 147 base pairs (bp) of DNA wrapped around the histone octamer (two copies each of histones H2A, H2B, H3, and H4) (Kornberg and Lorch 1999). Post-translational modifications (PTMs) such as acetylation and methylation of histones play important regulatory roles in almost every aspect of DNA metabolism, including transcription, replication, recombination, and DNA repair (Berger 2007; Downs et al. 2007; Groth et al. 2007; Kouzarides 2007). Among all of the modifications, histone acetylation at the promoter regions is a hallmark for an actively transcribed gene, and the level of acetylation

\footnotetext{
${ }^{4}$ Corresponding authors

E-mail yyshi@ustc.edu.cn

E-mail wujihui@ustc.edu.cn.

Article is online at http://www.genesdev.org/cgi/doi/10.1101/gad.188359.112.
}

is positively correlated with transcription rates (Liu et al. 2005; Pokholok et al. 2005; Rosaleny et al. 2007; Wang et al. 2008). The general mechanisms could be categorized into cis and trans. In a cis mechanism, histone acetylation is believed to relax chromatin via the neutralization of positive charges on lysines, thereby reducing the interaction between histones and DNA. On the other hand, a trans mechanism relies on the recruitment of epigenetic readers with various biological functions, as long as other effector proteins associated with them (Kouzarides 2007; Wang et al. 2007).

Monocytic leukemia zinc finger protein (MOZ, also referred to as KAT6A and MYST3) is a MYST family histone acetyltransferase (HAT) that catalyzes the transfer of an acetyl group from acetyl-CoA to the lysine $\varepsilon$-amino groups on histones, which plays roles in hematopoiesis (Thomas et al. 2006), skeletogenesis (Miller et al. 2004; Crump et al. 2006), and other developmental processes. 
It is a coactivator of various transcription factors (particularly for hematopoietic specificity) such as RUNX1 (AML1 [acute myeloid leukemia 1]) and PU.1 (Kitabayashi et al. 2001; Pelletier et al. 2002). MOZ was first identified in AMLs through its presence in reciprocal chromosomal translocations, leading to the fusion of MOZ to a transcription activator, CBP (Borrow et al. 1996); CBP-like coactivator p300 (Chaffanet et al. 2000); or a nuclear receptor coactivator, TIF2 (Carapeti et al. 1998). Each of these fusion proteins contains one or more HAT domains, indicating a role of aberrant acetylation in oncogenic transformation (Yang and Ullah 2007). Moreover, MOZ has been reported to be indispensible in the maintenance of hematopoietic stem cells and responsible for the differentiation of erythroid and myeloid cells (Katsumoto et al. 2006). Recently, another study in mouse embryos shows that MOZ is required for normal levels of H3K9 acetylation and gene expression at a large number of Hox loci and for identity specification of 19 body segments of the cervical and thoracic axial skeleton and nervous system (Voss et al. 2009).

In vivo, MOZ forms a tetrameric complex with ING5 (inhibitor of growth 5), EAF6 (Esa1-associated factor 6 ortholog), and the bromodomain PHD (plant homeodomain) finger proteins BRPF1, BRPF2, or BRPF3 to perform its functions (Ullah et al. 2008). As the catalytic subunit of the quartet complex, the biological consequences of MOZ depend on its localization to specific gene loci. Insight into how MOZ is recruited to these regions is important for disclosing the mechanisms underlying MOZ-mediated processes. MOZ is a multidomain protein containing a NEMM (N-terminal part of Enok, MOZ, or MORF) domain, a tandem PHD zinc finger, a MYST (HAT) domain for acetyl-transfer catalysis, a glutamate/aspartate (ED)-rich region, and a serine/methionine (SM)-rich region for transcriptional activation (Fig. 1A; Kitabayashi et al. 2001). The PHD zinc fingers are the only putative histone recognition motif capable of "reading" various histone modifications and unmodified histone tails (Martin et al. 2006; Palacios et al. 2006; Pena et al. 2006; Shi et al. 2006; Taverna et al. 2006; Mansfield et al. 2011), and this unique ability suggests its potential responsibility for the localization of MOZ on chromatin. Moreover, the tandem PHD finger only exists in MOZ/MORF and their orthologs among all of the HATs, shedding light on its special role for MOZ-mediated processes. However, little is known about the tandem PHD fingers; therefore, we were curious to investigate its structure and the corresponding functions. In this study, we defined the tandem PHD finger of MOZ (PHD12) as a histone $\mathrm{H} 3$ tail-binding module recognizing unmodified arginine residue 2 (H3R2) and acetylated lysine residue 14 (H3K14ac). The solution structure of PHD12 in a free state and the $1.47 \AA$ resolution crystal structure in complex with a H3K14ac peptide revealed the structural basis for the recognition, which was further supported by the subsequent chemical shift perturbation and mutagenesis experiments. Although the interactions between PHD12 and histone $\mathrm{H} 3$ do not affect the HAT activity of the MOZ MYST domain in vitro, the results of chromatin immunopre- cipitation (ChIP) assays indicated its contribution to targeting MOZ onto the HOXA9 gene promoter in vivo, concomitant with an enhanced local histone H3K14 acetylation and up-regulated HOXA9 transcripts. Our findings suggested that the recognition of H3R2/K14ac by PHD12 in MOZ might represent an important mechanism for its localization to and transcriptional activation of specific genes; on the other hand, our findings provided a clue of cross-talk between MOZ complex and various histone modifications within the $\mathrm{H} 3$ tails.

\section{Results}

PHD12 recognizes unmodified $R 2$ and acetylated K14 on histone $\mathrm{H3}$

Bromodomain has long been the only protein domain ever reported to recognize acetyl-lysine residues on proteins, such as GCN5 bromodomain recognizing acetylated Lys16 on histone $\mathrm{H} 4$ (Owen et al. 2000), and P/CAF bromodomain binding to lysine-acetylated peptides derived from major acetylation sites on histones H3 or H4 (Dhalluin et al. 1999), until recent studies reported the binding of the tandem PHD finger in D4 protein family member $3 \mathrm{~b}$ (DPF3b) to acetylated histones (Lange et al. 2008; Zeng et al. 2010). As the sequence of PHD12 in MOZ is highly similar to that of DPF3b, it is conceivable that MOZ PHD12 is also a candidate for acetylated histone recognition. To address this issue and beyond, we examined the binding affinity of MOZ PHD12 to a collection of histone peptides with or without modifications by NMR (nuclear magnetic resonance) and ITC (isothermal titration calorimetry). The results showed that MOZ PHD12 binds specifically to histone $\mathrm{H} 3$ with Lys14 acetylated (residues 1-18) (Fig. 1B,C), but not H4 with Lys16 acetylated (residues 11-21) (Supplemental Fig. S1), which is slightly different from that of DPF3b. Shortening the H3 tail (residues 1-8) or removing acetylation on H3K14 reduced binding affinity compared with the H3K14ac peptide (residues 1-18) (Fig. 1C; Supplemental Fig. S2), indicating that acetylation of Lys 14 could strengthen the interactions between histone $\mathrm{H} 3$ and PHD12. Moreover, trimethylation of $\mathrm{H} 3 \mathrm{~K} 4$ impedes the binding, while acetylation or trimethylation of $\mathrm{H} 3 \mathrm{~K} 9$ did not show a significant effect (Fig. 1C; Supplemental Fig. S3). In contrast, methylation of H3R2 devastates the binding completely (Fig. 1D), indicating the essentiality of H3R2 in this binding process. Taken together, these findings suggest that among various histone modifications, the tandem PHD finger of MOZ preferentially recognizes unmodified R2 and acetylated K14 on histone H3 tails.

Considering this bispecificity as independent events, we were curious to study them one by one to figure out how much each site contributes to the overall recognition specificity. Interestingly, although $\mathrm{H} 3(1-18)$ is a stronger substrate than H3 (1-8) (Fig. 1C; Supplemental Fig. S2), H3 (9-18) alone does not suffice to induce detectable binding, which could not be rescued by Lys 14 acetylation on this fragment [H3 (9-18)K14ac] (Fig. 1C; Supplemental Fig. S6). Likewise, the destructive effect of alanine sub- 
Qiu et al.

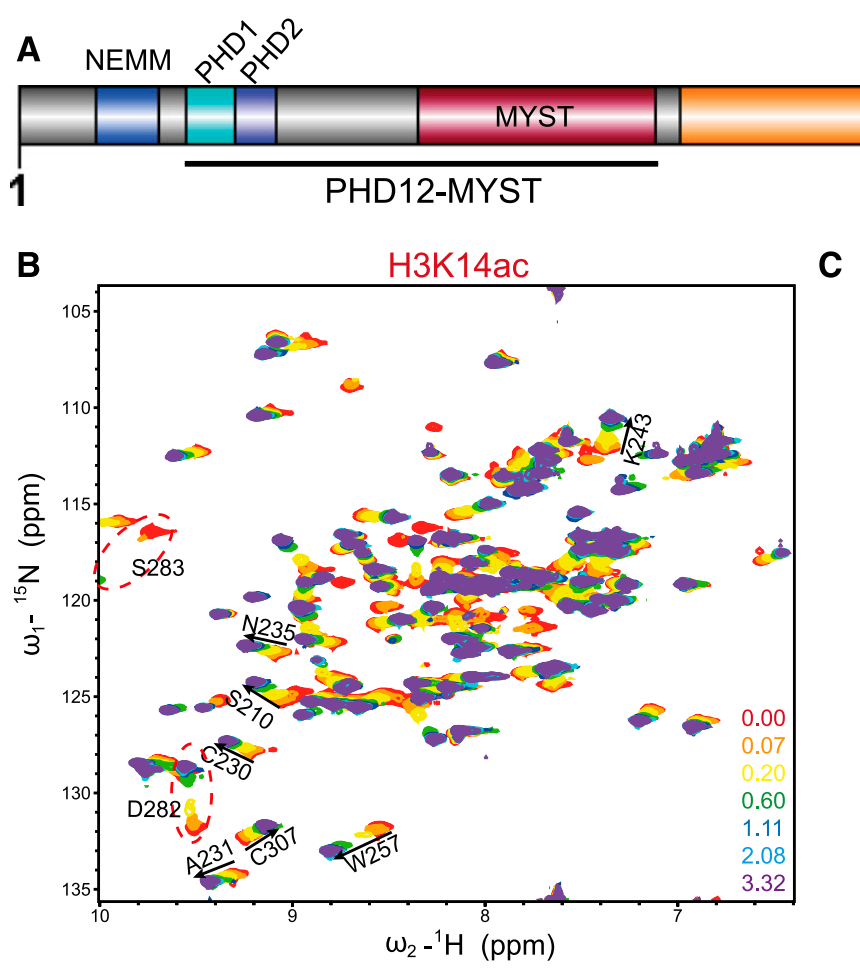

ED

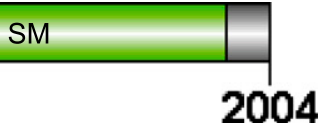

C

\begin{tabular}{ll}
\hline Histone Peptide & Kd value $(\mu \mathrm{M})$ \\
\hline unH3(1-18) & $64.9 \pm 4.6$ \\
H3(1-18)K4me3 & $286.5 \pm 46.1$ \\
\hline H3(1-18)K9ac & $61.3 \pm 2.6$ \\
H3(1-18)K9me3 & $55.6 \pm 12.5$ \\
H3(1-18)K14ac & $23.3 \pm 2.5$ \\
H3(1-18)K9acK14ac & $28.6 \pm 2.8$ \\
\hline H3(1-18)K14ac_T11A & $55.9 \pm 9.5$ \\
H3(1-18)K14ac_R2A & N/D \\
H3(1-18)R2me2a & N/D \\
\hline H3(1-18)R2me2s & N/D \\
\hline unH3(1-8) & $588.2 \pm 80.3$ \\
unH3(9-18) & N/D \\
H3(9-18)K14ac & N/D \\
\hline
\end{tabular}

D
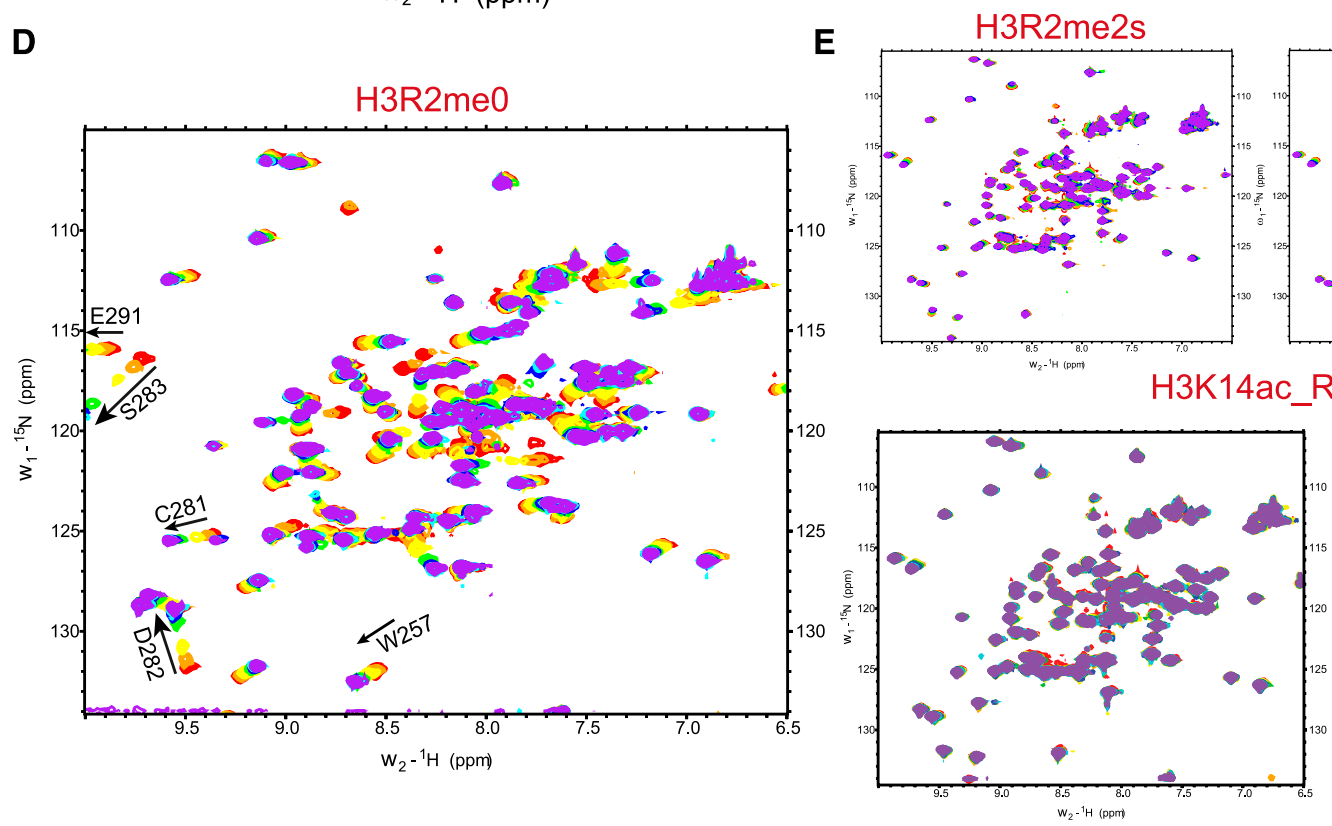

H3R2me2a
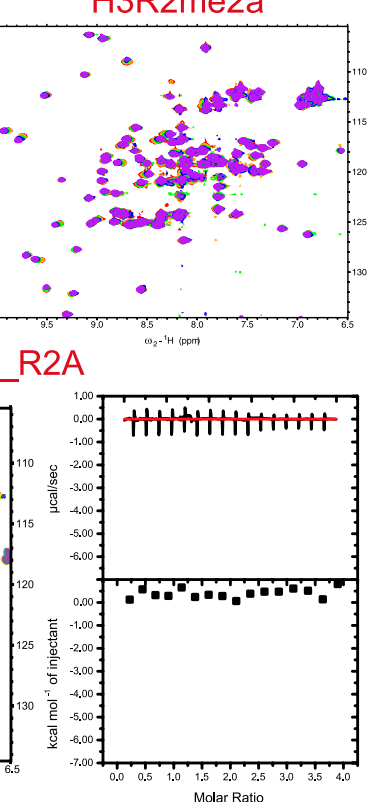

Figure 1. PHD12 recognizes unmodified R2 and acetylated K14 on histone H3. (A) Schematic representation of the domain structure of human MOZ. Domains are labeled as follows: (NEMM) N-terminal part of Enok, MOZ, or MORF; (PHD) plant homeodomain-linked zinc finger; (ED) glutamate/aspartate-rich region; (SM) serine/methionine-rich domain. (B) Seven superimposed ${ }^{1} \mathrm{H}^{15} \mathrm{~N}$ HSQC spectra of PHD12 (0.3 mM), collected during titration of the H3K14ac peptide, are color-coded according to the molar ratio of peptide PHD12. The red dashed circles indicate the residues with intermediate or slow exchange properties. $(C)$ Dissociation constants of the interactions between PHD12 and histone $\mathrm{H} 3$ tail peptides determined by ITC experiments. $(D)$ Superimposed ${ }^{1} \mathrm{H}^{-15} \mathrm{~N} \mathrm{HSQC}^{15} \mathrm{Hpctra}$ of PHD12 collected during titration of unH3 (1-18). (E, top) Superimposed ${ }^{1} \mathrm{H}_{-}{ }^{15} \mathrm{~N}$ HSQC spectra of PHD12 collected during titration of H3 (1-18)R2me2s or H3 (1-18)R2me2a peptide from left to right, respectively. (Bottom) Chemical shift perturbations in the twodimensional ${ }^{1} \mathrm{H}^{15} \mathrm{~N}$ HSQC spectra and ITC curves introduced by the titration of H3 (1-18)K14ac_R2A.

stitution of $\mathrm{R} 2$ is also not able to be reversed by a single acetylation on Lys14 [H3 (1-18)K14ac_R2A] (Fig. 1E). Therefore, in all respects, only when the hydrogen bonds responsible for $\mathrm{R} 2$ recognition remain intact could $\mathrm{PHD} 12$ show a preference for acetylated K14 to an unmodified H3 peptide. 
Overall structures of PHD12 in the free and H3K14ac-bound states

To unveil the mechanism of this combinatorial readout, we solved the solution structure of MOZ PHD12 in a free state (Protein Data Bank [PDB] ID 2LNO) (Fig. 2A) and the crystal structure of that in complex with a histone $\mathrm{H} 3$ (1-18) peptide containing R2me0 and K14ac (PDB ID 3V43) (Fig. 2B-D) with structural statistics listed in Supplemental Tables S1 and S2, respectively. The structures showed that each PHD finger adopts a canonical PHD finger fold, which consists of a two-strand anti-parallel $\beta$ sheet followed by a C-terminal $\alpha$ helix and two zinc atoms coordinated by the Cys4-His-Cys3 motif (Fig. 2B). In each structure, two PHD fingers associate with one another intimately in a face-to-back manner through the interactions between Glu247 and Arg251 in $\alpha 1$ from the first PHD finger (PHD1) and through the interactions between Ser283 and Arg286 in the $\beta 3-\beta 4$ sheet from the second
PHD finger (PHD2). In detail, via the carboxyl and carbonyl oxygen of Glu247, PHD1 forms two hydrogen bonds with two water molecules, bridging the polar contact to PHD2 through the hydroxyl and carbonyl oxygen of Ser283. Similar to the oxygen-mediated hydrogen bond network, a nitrogen atom on the side chain of Arg251 forms a pair of hydrogen bonds with another two water molecules, connecting the two amino nitrogen atoms on the Arg286 side chain (Fig. 2C). These polar interactions immobilize these two PHD fingers to a relatively stationary orientation, making them an integrated structural unit.

\section{PHD2 specifically recognizes unmodified R2 on histone H3}

Further analysis of the $1.47 \AA$ resolution complex structure revealed the structural basis for site-specific recognition of unmodified R2 on histone $\mathrm{H} 3$ by PHD2. As shown in Figure 2D, the first four residues of the bound
A
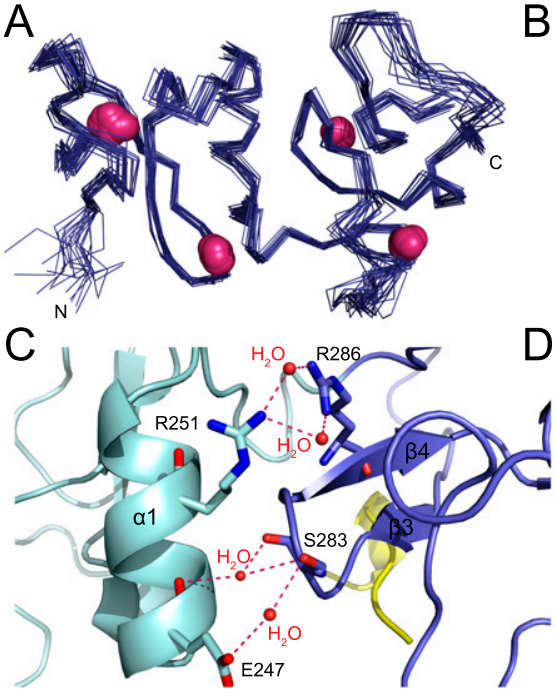

$\mathrm{E}$

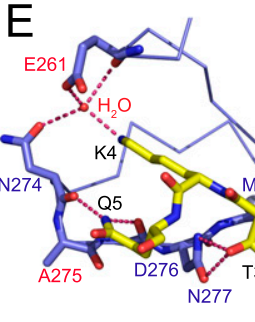

G

\begin{tabular}{ll}
\hline MOZ PHD12 & Kd value $(\mu \mathrm{M})$ \\
\hline WT & $19.1 \pm 3.0$ \\
S210A & $52.1 \pm 12.2$ \\
N235A & $184.2 \pm 84.6$ \\
S210D+N235R & $21.4 \pm 1.2^{*}$ \\
E261A & $85.8 \pm 23.1$ \\
A275D & $5.2 \pm 0.8$ \\
D282A & Not detected \\
\hline
\end{tabular}

B

D
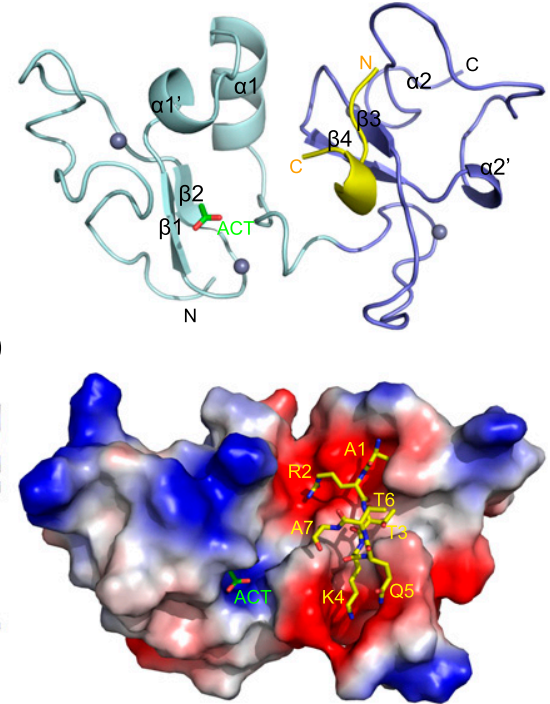

$\mathrm{F}$
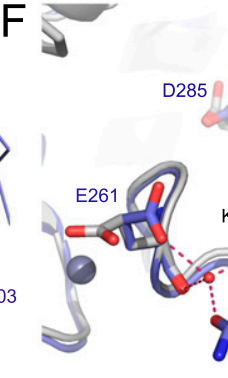

$\mathrm{H}$

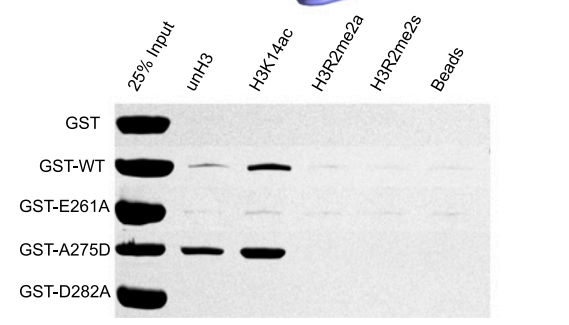

Figure 2. Structures of PHD12 in the free and H3K14ac-bound states. (A) Ensemble of the 20 lowest-energy structures for residues 204-313 of PHD12; the zinc atoms are highlighted as purple spheres. (B) Cartoon representation of the $1.47 \AA$ crystal structure of PHD12 in H3K14ac-bound states. PHD1 and PHD2 are colored in cyan and blue, respectively. The histone $\mathrm{H} 3$ peptide (yellow) and the bound acetate (green) are shown as sticks. (C) PHD1 and PHD2 associate with each other through a series of hydrogen bonds between $\alpha 1$ and the $\beta 3-\beta 4$ sheet. Intermolecular interactions are depicted as magentacolored dashed lines. A bridging water molecule involved in intermolecular recognition is shown as a red sphere. $(D, E)$ PHD12 recognition of the $\mathrm{N}$-terminal residues of the $\mathrm{H} 3 \mathrm{~K} 14 \mathrm{ac}$ peptide, depicted in a protein electrostatic potential surface $(D)$ and ribbon representation $(E)$. The bound $\mathrm{H} 3$ peptide and acetate are colored in yellow and green, respectively, with interacting residues on PHD12 colored in blue. $(F)$ Cartoon representation of the superposition of the structures of PHD12 in the free (silver) and H3K14ac peptide-bound (blue) states. The H3 (1-5) segment of the bound peptide is shown in yellow. $(G)$ Dissociation constants of the interaction between PHD12 or mutants and H3K14ac peptides determined by FPA (fluorescence polarization assay) using a C-terminal fluorescein isothiocyanate (FITC)-labeled H3K14ac peptide (residues 1-18) or ITC (indicated with asterisk). (H) In vitro peptide pull-down assays using various biotinylated histone peptides containing the indicated modifications. GST-PHD12 or the mutants were incubated with biotinylated histone peptides immobilized onto streptavidin Sepharose beads. Bound proteins were subjected to SDS-PAGE and stained by Coomassie blue. 
H3 peptide are embedded extendedly into the acid groove on the surface of PHD2. More precisely, the $\mathrm{N}$ terminus of the peptide is tethered to the main chain carbonyl oxygen of Gly303, and the side chain of Ala1 in the H3 peptide is buried within a hydrophobic cage formed by residues Met300, Pro301, and Trp305. Located near a patch of negatively charged area, the side chain of Arg2 forms extensive hydrogen bonds with the oxygen atoms on the side chain of Asp282 and Asp285, both of which reside in the hairpin linking $\beta 1$ and $\beta 2$ of PHD2. In addition, the $\mathrm{N}_{\eta} 2 \mathrm{H}$ proton of Arg2 also forms a polar contact with the backbone carbonyl oxygen of Cys281. It is the network of these five hydrogen bonds that stringently guarantees the specific recognition of the H3R2 mark by PHD2. From the structure, we speculated that methylation of R2 will impede the formation of the hydrogen bond network, thus disabling the interaction. Expectedly, the binding of PHD12 to H3 peptide was totally abolished when Arg2 is either symmetrically or asymmetrically dimethylated (Fig. 1E). Besides these, many other polar contacts were observed between PHD2 and the bound H3 peptide: The hydroxyl oxygen of Thr3 interacts with the acylamide group of Asn277; the backbone nitrogen of Lys4 interacts with the carbonyl oxygen of Met278; the $N_{\eta}$ nitrogen of Lys4 forms hydrogen bonds with three oxygen atoms from Glu261 and Asn274, mediated by an ordered water molecule; and the acylamide nitrogen of Gln5 interacts with two carbonyl oxygens from the main chain of Asn274 and Asp276, respectively (Fig. 2E). These interactions, together with the hydrogen bond network between PHD2 and the $\mathrm{N}$ terminus of the $\mathrm{H} 3$ peptide, demonstrate at atomic resolution how the recognition of $\mathrm{R} 2$ in a $\mathrm{H} 3$ sequence context is guaranteed by PHD2.

To further confirm the role of R2 in H3 binding, we used an H3 (1-18)K14ac peptide containing a R2A mutation to titrate PHD12 and monitored it by ITC and NMR, respectively. The results showed no apparent binding when Arg2 was mutated to Ala (Fig. 1E), recapitulating the destructive effect of R2 methylation. Accordingly, several point mutations on PHD2 were prepared: E261A or D282A was designed to disrupt the hydrogen bond formation with Lys4 or Arg2, respectively; while A275D was expected to form an extra polar contact between the residue 275 and Gln5 (Fig. 2E). The FPA (fluorescence polarization assay) results showed that the E261A mutation decreased the binding affinity to the H3K14ac peptide about fourfold to fivefold, and the D282A mutation totally abolished binding, while, on the other hand, the A275D mutant strengthened this binding with a disassociation constant decrease of threefold to fourfold (Fig. 2G). A similar result was obtained from a peptide pull-down assay (Fig. 2H), which further supports the proposed structural basis for H3R2/K4 recognition by PHD2.

From the structure comparison of MOZ PHD12 in the free and H3-bound states (Fig. 2F), the backbones of the two structures are superimposed strictly with a root-meansquare deviation (r.m.s.d.) of $0.77 \AA$, whereas a conformational change was observed on the segment from Asp269 to Asn 277 upon $\mathrm{H} 3$ peptide binding. In the free state, this segment folds into a short loop followed by a small $\alpha$ helix, while in the peptide-bound state, it is stretched to form a longer loop to accommodate the side chains of Lys4 and Gln5 (Fig. 2F). The NMR relaxation parameters showed that the segment between Gly272 and Asn277 is relatively flexible (Supplemental Fig. S5), suggesting an "induced fit" of PHD2 during the peptide-binding process.

\section{PHD1 is a histone H3 acetylated Lys14-binding module}

Unexpectedly, in the complex structure, instead of acetylated Lys14, an acetate group from the solvent was observed at the acetyl-binding site in PHD1 (Fig. 3C). Furthermore, a side chain of arginine from another PHD12 molecule in the same unit cell occupied the space above the acetate group (Supplemental Fig. S4). Therefore, the competition of acetate in solvent and crystal packing force made us fail to obtain the electron density map of the acetylated Lys14 in the H3K14ac-bound state. In order to investigate the structural basis for acetylated Lys14 recognition by PHD12, a NMR chemical shift perturbation experiment was employed with two histone $\mathrm{H} 3$ tail peptides in different Lys14 acetylation states. The comparison between chemical shift changes induced by the addition of different $\mathrm{H} 3$ peptides helped us map the acetylated Lys14binding site on MOZ PHD1. The results summarized in Figure 3A indicated that the overall chemical shift perturbation introduced by $\mathrm{H} 3(1-18) \mathrm{K} 14 \mathrm{ac}$ is larger than that introduced by $\mathrm{H} 3(1-18)$, and the ITC data shown in Figure $3 \mathrm{~B}$ are consistent with this. Several residues around Ser210 and Asn235 exhibited large chemical shift changes when titrated by $\mathrm{H} 3$ (1-18)K14ac (enclosed by red circles in Fig. $3 \mathrm{~A}, \mathrm{C})$ without being affected by unmodified H3 (1-18), indicating that Ser210 and Asn235 play specific roles in the acetylated Lys14 recognition. Taken together, MOZ $\mathrm{PHD} 1$ recognizes acetylated Lys14 on $\mathrm{H} 3$ through the collaborative contributions of Ser210, Asn235, and the other residues around them.

In a recent work, Zeng et al. (2010) reported the molecular mechanism underlying the sequence-specific recognition of H3K14ac by DPF3b tandem PHD finger as a functional unit. A DALI (Holm and Rosenstrom 2010) search using MOZ PHD12 as query structure indicated that the tandem PHD finger of DPF3b is the one with the highest structural similarity in all PDB entries, with a $\mathrm{C} \alpha$ r.m.s.d. of $1.9 \AA$ and a $Z$-score of 13.0. Based on the structural similarity and the same binding substrate, a structural comparison between the tandem PHD fingers of MOZ and DPF3b (Fig. 3D) would be very informative to determine the acetylated Lys14-binding sites on MOZ, which are otherwise invisible in our complex structure. As is demonstrated in the complex structure of DPF3b with the H3K14ac peptide, the methyl group of the acetylated Lys14 inserts into a hydrophobic pocket formed by residues Phe264, Leu296, Trp311, and Ile314. The corresponding hydrophobic residues on MOZ are Phe211, Leu242, Trp257, and Ile260, which together indeed make up a conserved hydrophobic pocket. Moreover, in the DPF3b complex, the rim of the binding pocket is clustered with Asp263, Arg289, and zinc-coordinating Cys313, with the 
Downloaded from genesdev.cshlp.org on April 26, 2023 - Published by Cold Spring Harbor Laboratory Press

A

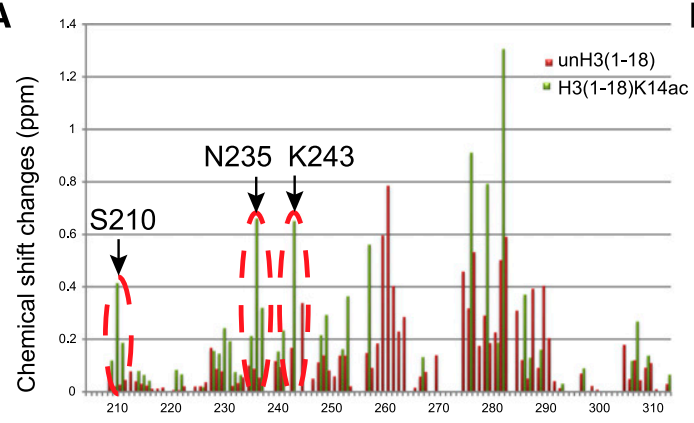

B

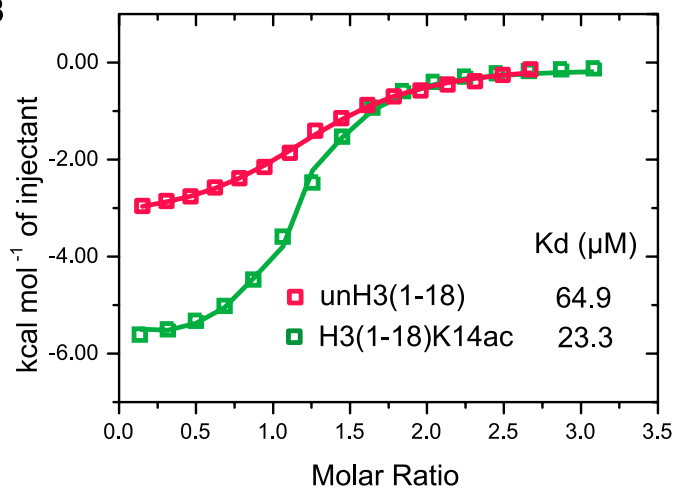

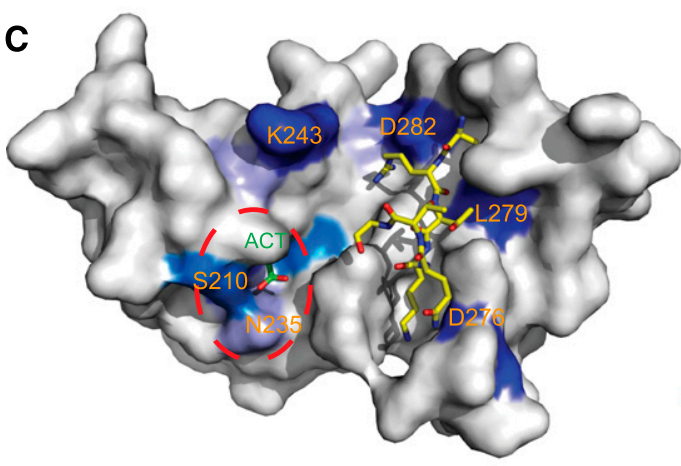

E

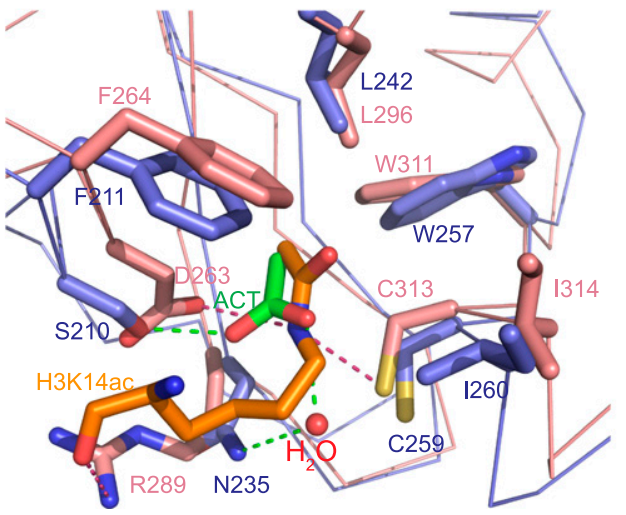

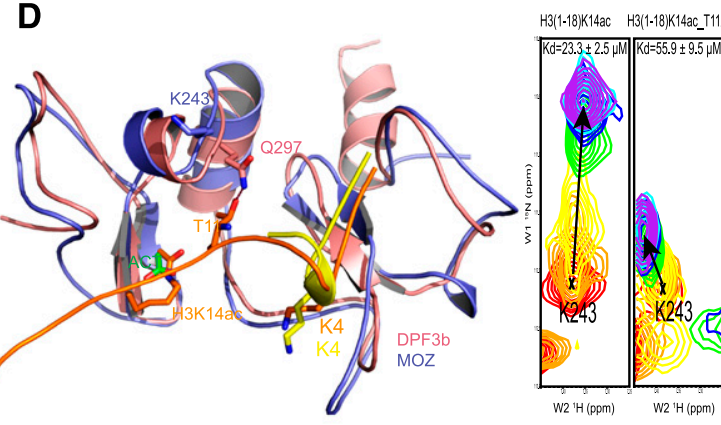

$\mathbf{F}$

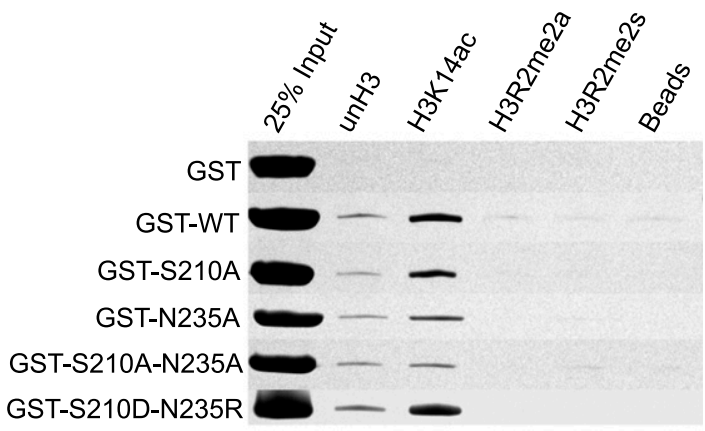

G

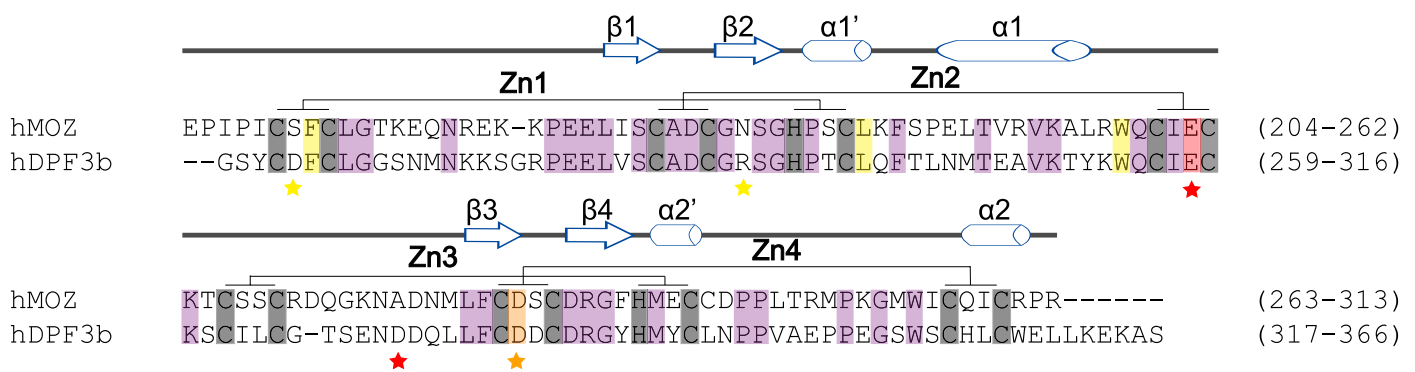

Figure 3. $\mathrm{MOZ} \mathrm{PHD} 1$ is a histone $\mathrm{H} 3$ acetylated Lys14-binding module. $(A)$ The histogram displays ${ }^{1} \mathrm{H},{ }^{15} \mathrm{~N}$ chemical shift changes caused by H3 (1-18) (red) or H3 (1-18)K14ac (green) peptide binding. Residues S210, N235, and K243 are circled by red dashed lines. (B) Superposed exothermic ITC enthalpy plots for the binding of PHD12 to H3 (1-18) and H3 (1-18)K14ac peptides. The insert lists the measured binding constants. $(C)$ The molecular surface of PHD12 with residues colored according to the magnitude of the observed chemical shift change upon addition of H3K14ac peptide. The surface residues are color-coded according to the following scheme: (blue) $\Delta \delta>0.6$; (marine) $0.6>\Delta \delta>0.4$; (light purple) $0.4>\Delta \delta>0.2$. The positions of residues S210, N235, K243, D276, L279, and D282 are shown. The bound peptide (yellow) and acetate group (green) are shown in sticks. The red dashed circle in $C$ encloses the residues in the first PHD finger that are involved in acetylated lysine recognition. ( $D$, left) Superimposition of the structures of tandem PHD fingers of MOZ (blue) and DPF3b (salmon) in complex with H3K14ac peptides (yellow in the MOZ complex and orange in the DPF3b complex). (Right) Chemical shift perturbation of residue Lys243 showing that Lys 243 takes a role in the interaction with T11 on histone H3K14ac peptide. (E) Magnified view of the superimposed acetylated lysine-binding pocket of MOZ (blue) and DPF3b (salmon), with key interacting residues labeled. (F) In vitro peptide pull-down assays like that in Figure 2H. $(G)$ Sequence alignment of tandem PHD fingers of MOZ and DPF3b. Sequence numbers are shown at the right of the corresponding protein sequences. Highly conserved residues in the PHD fingers are highlighted with a purple background. Zinccoordinated cysteine and histidine residues are grouped by a gray background. Residues responsible for interacting with $\mathrm{K} 14 \mathrm{ac}$, $\mathrm{K} 4 \mathrm{me} 3$, and unmodified R2 are highlighted in yellow, red, and orange, respectively. Stars mark residues subject to the mutational analysis in this study. 
acetyl amide of H3K14ac forming a pair of hydrogen bonds to the carboxyl oxygen of Asp263 and the sulfur of Cys313, whereas the backbone acylamide oxygen of Lys 14 is involved in another hydrogen bond with the Arg289 side chain. Although the Asp263 and Arg289 at the hole of the binding pocket in DPF3b change to a Ser210 and an Asn235 in MOZ (Fig. 3G), water-mediated hydrogen bonds between the carboxyl oxygen of the acetate and Ser210 hydroxyl oxygen as well as Asn235 amino nitrogen were observed in the complex structure (Fig. 3E). These interactions stabilize the acetate group and indicate the possibility of the acetyl amide of H3K14ac forming hydrogen bonds with Ser210 and Asn235 in a similar manner. Supporting this, alanine substitution of Ser210 or Asn235 causes a reduction in the binding affinity of PHD12 to the H3K14ac peptide (Fig. 2G), and the double mutation destroys the K14ac binding almost entirely (Fig. 3F). Conversely, we also designed a double point mutation of S210D and N235R on MOZ, interested in the binding properties when these two key residues are identical to the corresponding residues of DPF3b. A further ITC-binding assay showed an almost unaffected K14ac binding of this mutant (Figs. 2G, 3F), indicating that S210 and N235 in $\mathrm{MOZ}$ are very likely to suffice for K14ac binding compared with their aspartate and arginine counterparts in DPF3b. In summary, these results indicate that MOZ recognizes the acetylated Lys14 by a hydrophobic pocket and polar residues in PHD1, which is similar to that in DPF3b.

Besides the K14ac-binding site, there are two major differences between the tandem PHD fingers of MOZ and DPF3b from a global aspect. The first is that the C-terminal $\alpha$ helix in DPF3b PHD2 changes to a short loop owing to a proline residue. The second is that the loop ahead of the first $\beta$ strand of MOZ PHD2 is longer than that of DPF3b PHD2 (Fig. 3D), making it possible to accommodate the trimethylation of Lys4 on histone $\mathrm{H} 3$ (Fig. 1C; Supplemental Fig. S3).

Aiming to determine the contribution of MOZ PHD1 to the histone $\mathrm{H} 3$ binding directly, we analyzed peptides comprising only the portion of the $\mathrm{H} 3$ tail that binds to PHD1 with and without acetylated K14, as has been mentioned before. As shown in Figure 1C and Supplemental Figure S6, however, histone H3 peptides do not bind to PHD12 without the $\mathrm{N}$-terminal eight residues; even Lys 14 was acetylated, indicating the indispensable role of the $\mathrm{N}$-terminal residues of $\mathrm{H} 3$ peptides in the binding process. In fact, as methylation and alanine mutation on R2 abolish binding completely (Fig. 1E), it is very unlikely for PHD12 to show any affinity to an H3 peptide construct like unH3 (9-18) without unmodified R2.

Considering the weak binding of acetylated Lys14, we were curious which residues on $\mathrm{H} 3$ around $\mathrm{K} 14$ were also involved to guarantee the Lys14 specificity. The structural comparison with the DPF3b-H3K14ac complex indicated $\mathrm{T} 11$ on histone $\mathrm{H} 3$ as the only residue with the potential to also be involved in binding (Fig. 3D). Therefore, we designed the T11A mutation on histone $\mathrm{H} 3$ peptide and then measured its binding affinity to PHD12 by ITC and NMR. The ITC data showed a reduction in overall binding, with the $\mathrm{K}_{\mathrm{d}}$ value rising from $23.3 \pm 2.5 \mu \mathrm{M}$ to $55.9 \pm 9.5 \mu \mathrm{M}$
(Fig. 1C). In addition, K243 demonstrated an apparent discrepancy in chemical shift changes upon H3 and H3T11A binding, indicating its role in tethering T11 to histone $\mathrm{H} 3$ and therefore optimizing the binding environment of acetylated K14 (Fig. 3D, right). By combining the NMR titration results with the ITC data, we could conclude that the residues surrounding K14 may contribute to the H3 peptide binding to $\mathrm{PHD12}$, which points to a scenario where instead of recognizing acetylated lysine per se, $\mathrm{PHD} 12$ is capable of selecting K14ac in a sequence-specific manner.

\section{PHD12 binding to histone does not affect the HAT activity of $M O Z$ in vitro}

MOZ (as a HAT), its HAT activity, and its substrate specificity are under strict regulation by other components of the MOZ/MORF complex. Both BRPF1 and ING5 have been shown to up-regulate its acetyltransferase activity (Ullah et al. 2008), and the presence of full-length ING5 was further reported to result in preferential acetylation of methylated H3K4 histone peptides (Champagne et al. 2008). In another report, the PHD finger ahead of a demethylated Jumonji domain in PHF8 not only directs the substrate specificity of PHF8, but also increases its enzymatic activity by 12 -fold (Horton et al. 2010). Since PHD12 is the only histone recognition module in MOZ, whether its interaction with histone $\mathrm{H} 3$ also affects the HAT activity is an interesting question. To address this issue, a truncated MOZ fragment comprising the MYST domain, which is responsible for HAT activity; PHD12-linkerMYST (Fig. 1A); mutant PHD12 (D282A)-linker-MYST; and a PHD12 control were constructed with a C-terminal His tag and expressed in Escherichia coli, respectively. After affinity purification, their enzymatic activities were monitored based on an ${ }^{3} \mathrm{H}$-based radioactive HAT assay using excessive recombinant histone octamers as substrate. However, although the instability of the PHD12linker-MYST protein led to long error bars, MYST, wildtype PHD12-linker-MYST, and the mutant all exhibited similar HAT activities against all of the histones compared with the PHD12 control (Supplemental Fig. S7A,B). By the same token, with the D282A mutant, a HAT assay using recombinant histones containing a H3R2A mutation as substrate was performed. Compared with the wild-type histone octamers, the mutant substrate hardly showed any difference in HAT activity (Supplemental Fig. S7C,D). All of the above experiments combined indicated that the HAT activity is independent of PHD2 binding to histone $\mathrm{H} 3$ at the histone octamer level. The same conclusion could also be made from the competition experiment, in which the excessive $\mathrm{H} 3$ (1-18)K14ac peptide was added to the recombinant histone octamers while its HAT activity was monitored (Supplemental Fig. S7E). As the ITC data revealed no direct interdomain physical contact between PHD12 and the MYST domain (Supplemental Fig. S8), it is likely that PHD12 would never directly affect the enzymatic activity of the MYST domain.

Histone octamers might represent a poor substrate to study whether the potential association of PHD fingers 
with acetylated histones could influence HAT activity because we cannot rule out the possibility that these two adjacent domains work on different nucleosomes. In order to better represent its native substrate, we performed the HAT assay with oligonucleosomes extracted from HeLa cells, which are modified and thus contain the ideal binding motif for the PHD fingers. However, we failed to detect any HAT activities of either PHD12-linker-MYST or the MYST domain only (Supplemental Fig. S7F). A probable explanation rests on the requirement of whole-complex integrity, which is consistent with the previous report that MOZ/MORF HAT activity against oligonucleosomes needs BRPF1 and ING5 (Ullah et al. 2008).

\section{PHD12 associates with histone H3K14ac in vivo}

To assess the interaction of MOZ PHD12 with histone H3K14ac in vivo, a coimmunoprecipitation assay was performed. Lysates from 293T cells expressing Flag-PHD12 were immunoprecipitated with anti-Flag M2 affinity gel followed by Western blot analysis with anti-H3 and antiH3K14ac antibodies, respectively. Compared with the plasmid control, Flag-PHD12 markedly bound to nucleosomes with H3K14ac modifications (Fig. 4A), indicating that MOZ PHD12 physically associates with the H3K14ac mark in vivo. Notably, the enrichment of acetylated H3K14 relative to the input is obviously less than that of the total H3, indicating that not all histone H3 in PHD12bound nucleosomes is acetylated at Lys14. As nucleosomes, which contain two copies of histone $\mathrm{H} 3$, most probably remained intact during our experiment procedures, these K14ac-free histone $\mathrm{H} 3$ proteins might interact with PHD12 through unmodified R2, although this speculation needs to be further confirmed. Furthermore, confocal fluorescence imaging demonstrated that the ectopically expressed Flag-MOZ fusion protein is colocalized with H3K14ac sites in the H1299 cell nucleus, which became much less apparent in the case of the acetyl-lysine-bindingdeficient mutant S210A (Fig. 4B). This could be additional evidence of the physiological association of MOZ PHD12 with H3K14ac in vivo.

\section{PHD12 contributes to the localization of MOZ on the HOXA9 promoter}

Previous studies in mice and zebrafish indicated the key role of MOZ in regulating HOX gene expression (Crump et al. 2006; Voss et al. 2009). Moreover, one recent study in HEK293T and CD44 ${ }^{+}$cells has demonstrated the binding of MOZ to the HOXA9 gene promoter (Paggetti et al.
2010). In light of our findings that the tandem PHD finger of MOZ could interact with the histone $\mathrm{H} 3$ tail, an interesting question emerges as to whether this binding contributes to a gene-specific targeting. Therefore, we carried out ChIP assays followed by quantitative PCR (ChIP-qPCR) using 293T cells with transient expression of MOZ in full length. As shown in Figure 5, A and B, exogenous MOZ was efficiently recruited to the promoter of HOXA9, but not to the promoter of HOXB1, HOXC5, and HOXD12.

Based on our structural information, we further tested the contributions of each domain of PHD12 in this targeting process by introducing two point mutations, S210A and D282A, as mentioned above, which disrupts its recognition of acetylated K14 and unmodified R2 on histone $\mathrm{H} 3$, respectively. The results showed that without affecting the expression level (Fig. 5C) and the nuclear localization of MOZ (Supplemental Fig. S9), the mutant S210A or D282A has a destructive effect on the localization of MOZ on the HOXA9 promoter by a reduction of $>50 \%$ (Fig. 5B,D). In these assays, the constant low levels of target gene in groups employing normal mouse IgG as a coimmunoprecipitation negative control indicates the high fidelity of our quantification data (Fig. 5D). Taken together, these results suggest the critical role of PHD12 for targeting MOZ to the promoter of HOXA9.

\section{MOZ PHD12 binding H3 is important for the HOXA9 promoter acetylation and HOXA9 transcription}

$\mathrm{MOZ}$ (as a HAT) is responsible for H3K9 or K14 acetylation (Doyon et al. 2006; Voss et al. 2009). As PHD12 functions to target MOZ to the HOXA9 promoter, it is conceivable to speculate that this PHD12-dependent enrichment could lead to enhanced promoter acetylation. To address this issue, we monitored the acetylation level of the HOXA9 promoter region by ChIP using antibodies against acetylated H3K9 and acetylated H3K14 followed by qPCR. Considering our previous failure in detecting any HAT activity against HeLa oligonucleosomes using a MOZ fragment alone in the absence of a complex and the reports that BRPF1 could greatly enhance its HAT activity (Doyon et al. 2006), we cotransfected GFP-BRPF1 with Flag-MOZ (wild type or mutants) into 293T cells to better preserve the enzymatic activity of the ectopically expressed MOZ. As shown in Figure 6A, 293T cells expressing wild-type MOZ, but not cells expressing the S210A or D282A mutant, exhibited a more than twofold increase in the levels of H3K14 acetylation on the HOXA9 promoter compared with control cells. Notably, the levels of H3K9 acetylation
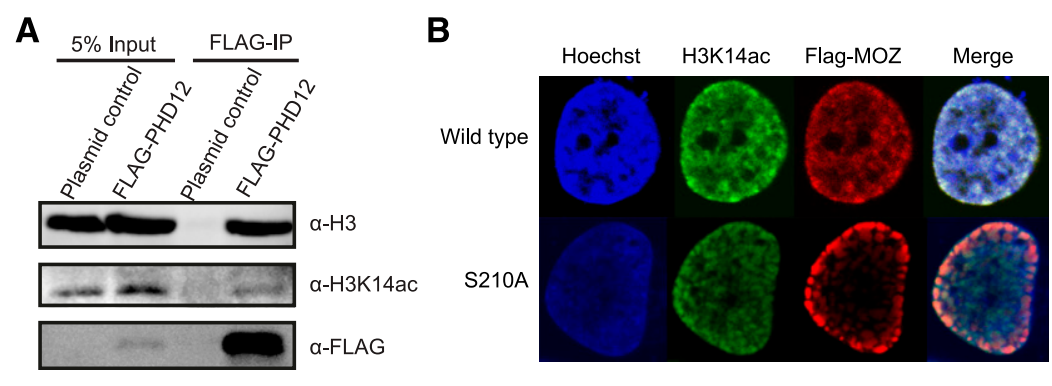

Figure 4. MOZ associates with histone H3K14ac in vivo. (A) Lysates from $293 \mathrm{~T}$ cells expressing Flag-tagged PHD12 or control vector were immunoprecipitated by anti-Flag M2 affinity gel followed by Western blot analysis with the indicated antibodies. (B) Colocalization of endogenous H3K14ac with ectopically expressed MOZ or its acetyllysine-binding-deficient mutant S210A was examined by immunofluorescence assay with the indicated antibodies in H1299 cells. 
Qiu et al.
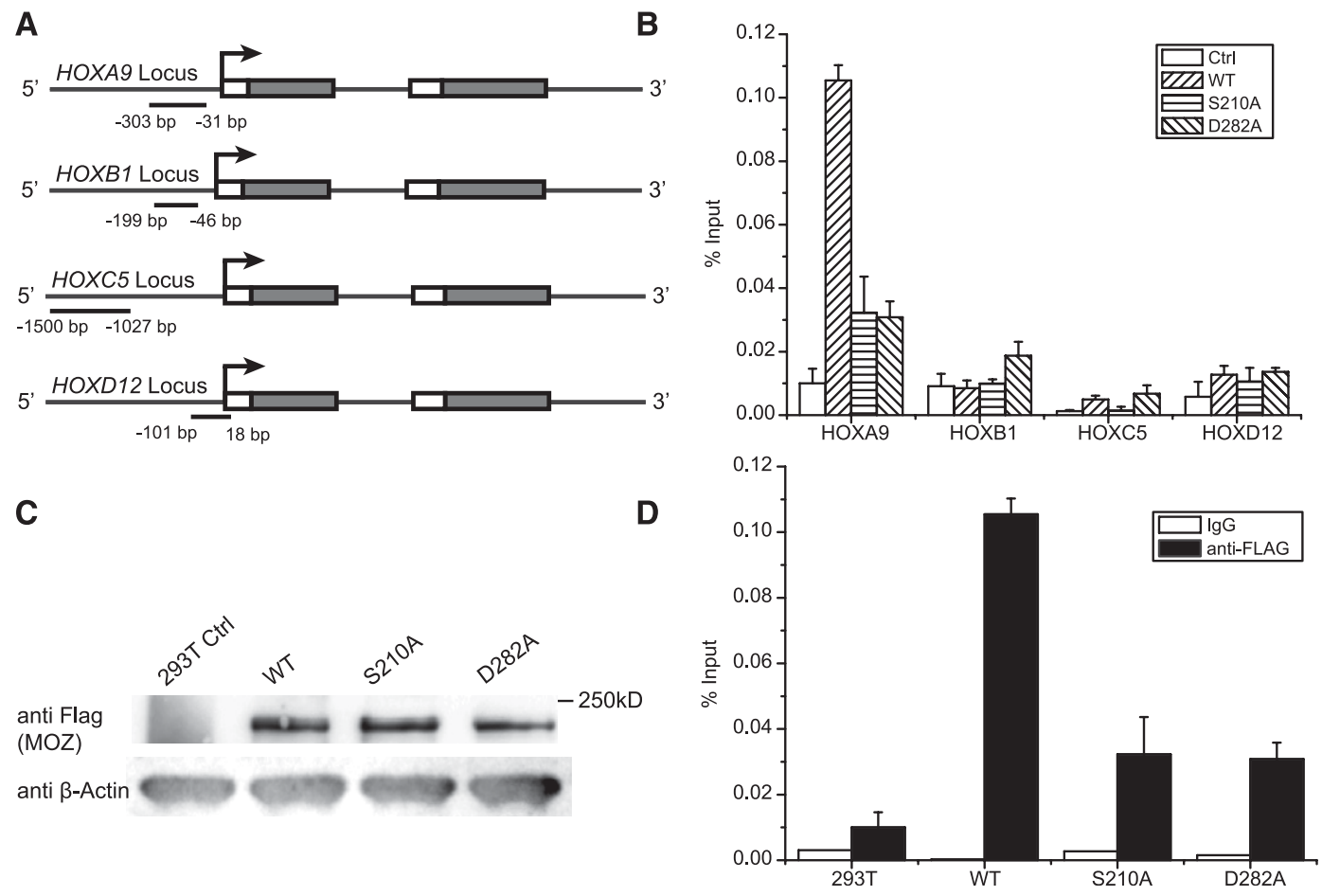

Figure 5. PHD12 contributes to the localization of MOZ on the HOXA9 promoter. (A) Schematic representation of parts of the HOXA9, HOXB1, HOXC5, and HOXD12 loci (not to scale). The positions of the primers used to reveal the ChIPs by PCR are indicated, relative to the HOXA9, HOXB1, HOXC5, and HOXD12 transcription start sites (arrows). (B) An average of three real-time PCR replicates of a representative experiment is displayed as a function of percentage of input signal, with error bars reflecting \pm SD of PCR product threshold error among the replicates. $(C)$ A Western blot with anti-Flag and anti- $\beta$-actin antibodies showed that all of the constructs were expressed at appropriate levels. (D) Coimmunoprecipitation control of normal mouse IgG compared with anti-Flag antibody group showed the high fidelity of our quantification data.

on the HOXA9 promoter remained similar in all of the cells expressing wild-type or mutant MOZ. These results indicate the $\mathrm{H} 3 \mathrm{~K} 14$-specific acetylation role of $\mathrm{MOZ}$ at the HOXA9 gene promoter. Combined with the previous ChIP data, these results provide a promising picture where the interaction between PHD12 and histone $\mathrm{H} 3$ helps MOZ promote the HOXA9 promoter acetylation, mainly at the K14 site. Meanwhile, consistent with previous reports (Voss et al. 2009; Mishima et al. 2011), we found that the global acetylation level remained unperturbed in the absence or presence of cotransfected BRPF1 (Fig. 6B), indicating that the enhancement of the acetylation level might be restricted to specific genes.

Generally, acetylation level is positively correlated with gene transcription. As the interaction between PHD12 and $\mathrm{H} 3$ is important for the HOXA9 promoter acetylation, we next examined whether this interaction functions in the HOXA9 gene transcription. As shown in Figure 6C, with the expression of wild-type MOZ, but not the S210A or S282A mutant, HOXA9 mRNA levels were significantly elevated by more than onefold. Based on these results, we could conclude that the interaction between MOZ PHD12 and histone $\mathrm{H} 3$ is important for the HOXA9 gene transcription.

On the other hand, under the condition of cotransfection of GFP-BRPF1 and Flag-MOZ (wild type or the S210 or D282 mutant), we analyzed the distribution of BRPF1 and
MOZ by fluorescence colocalization assays. Consistent with the previous reports (Laue et al. 2008; Ullah et al. 2008), the overlap of the fluorescence signal from GFP and immune probes against MOZ indicates that the spatial colocalization between MOZ and BRPF1 was unaffected by the mutations (Supplemental Fig. S10). As BRPF1 is shown to be the scaffold of the tetrameric complex and sufficient for the complex formation in vivo (Ullah et al. 2008), it is promising that the biological consequence that we witnessed in this study is largely under its native condition as a complex.

Overall, consistent with our in vitro findings, we identified the tandem PHD finger as a H3 unmodified R2 and acetylated K14 reader with a regulatory function in HOXA9 promoter acetylation and hence the gene transcription. Combined with our previous data, a mechanism could be proposed that through the recognition of the H3R2me0-H3K14ac mark by PHD12, MOZ could be guided onto the HOXA9 locus, where it acetylates adjacent histone and finally switches the HOXA9 gene to its active state.

\section{Discussion}

The tandem PHD finger is a functional unit

Extensive studies indicate that histone acetylation is an important epigenetic mark for gene transcription in a chro- 
A

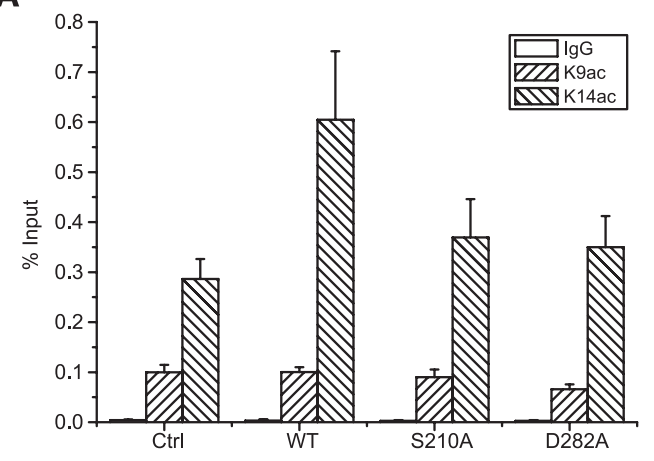

C

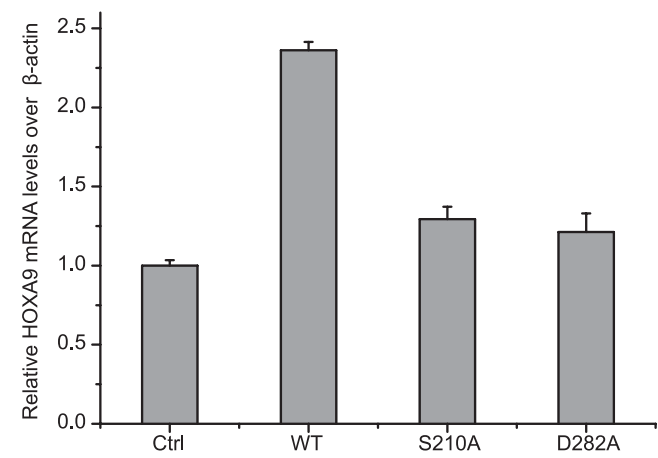

B

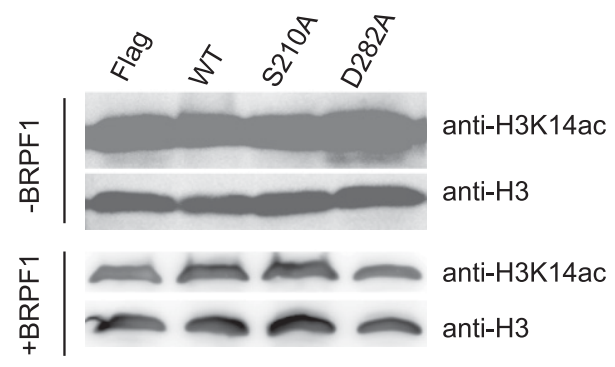

Figure 6. MOZ PHD12 binding of $\mathrm{H} 3$ is important for the HOXA9 promoter acetylation and HOXA9 transcription. (A) Lysates from 293 T cells expressing wild-type MOZ or the S210A or D282A mutant were subjected to ChIP assays with anti-K9ac and anti-K14ac antibodies, respectively, followed by qPCR analysis with $H O X A 9$ promoter-specific primers. The data are represented as mean \pm SD of three real-time PCR replicates. $(B)$ The global acetylation level of the cells in the absence or presence of cotransfected BRPF1, which was detected by Western blot analysis with the indicated antibodies. $(C)$ The relative mRNA levels in cells cotransfected with MOZ (wild type or mutant) and BRPF1, which were normalized by $\beta$-actins. The data are represented as mean \pm SD of three real-time PCR replicates.

matin context. Knowledge concerning effector modules capable of recognizing acetyl-lysine has long been limited to bromodomains. The tandem PHD finger of DPF3b, as a novel acetyl-lysine-binding module, was identified recently (Lange et al. 2008; Zeng et al. 2010). Due to the current limited information of the tandem PHD finger, it is necessary to further investigate its potential and underlying structural mechanism for specific recognition of acetyl-lysine. Our results show that the tandem PHD finger of MOZ is a histone recognition module that combinatorially reads unmodified histone H3R2 and acetylated H3K14. As described above, the two PHD fingers associate with each other intimately through numerous interactions, which define the two PHD fingers as an integral structural module.

Several nuclear proteins-such as DPF1/2/3b, MLL3, and PHF10 - containing the tandem PHD module were identified by the BLAST program using the PHD12 sequence as a query. The sequence alignment indicates that all individual PHD fingers in the modules adopt a canonical Cys4-His-Cys3 motif to coordinate zinc atoms. The two PHD fingers are connected by a short two-residue linker and form an integrate module (Supplemental Fig. S11). Residues that are involved in R2 and K4 recognition by the second PHD finger are conserved, except for the substitution of acid residue by alanine in MOZ, which might lead to the accommodation for methylation of K4. For acetylated K14 binding, a hydrophobic pocket comprised of three residues in the first PHD finger was conserved among all of these proteins; however, the residues responsible for hydrogen bond formation with the acetyl amide and the backbone acylamide oxygen of acetylated H3K14 are variable. In PHF10 and MLL3, the residues corresponding to Ser210 in MOZ change to hydrophobic residues, which might decrease or prevent their binding to acetylated lysine, indicating an unconserved role of the first PHD finger in histone recognition. Notably, both MLL3 and MOZ are histone-modifying enzymes responsible for $\mathrm{H} 3 \mathrm{~K} 4 \mathrm{me}$ and $\mathrm{H} 4 \mathrm{~K} 14 \mathrm{ac}$, respectively. Generally speaking, PHD fingers in this kind of protein regulate their chromatin localization and activity in a highly dynamic manner. In fact, tight binding of PHD fingers to histone might compromise the dynamic regulations by fixing the enzymes to chromatin, providing a possible interpretation of the weak binding of the tandem PHD fingers of MOZ and MLL3 to histone. Therefore, as a partially conserved functional unit, the tandem PHD finger deciphers the histone code to modifying enzymes, allowing them to function in a dynamic process. 
Unique recognition of unmodified histone H3K4 (H3K4meO) by PHD2 of MOZ

Previous studies have identified a group of PHD fingers characterized by binding to $\mathrm{H} 3 \mathrm{~K} 4 \mathrm{me} 0$, including those of a BRAF35-HDAC complex protein (BHC80), BRPF2, autoimmune regulator (AIRE), DNA (cytosine-5)-methyltransferase 3-like protein (DNMT3L), and DPF3b. The interactions underlying this type of recognition are generally mediated by direct hydrogen bonds between the oxygen atoms on a single or couple of conserved residues and the amino hydrogen on Lys4 (Supplemental Fig. S12). These conserved residues contain negatively charged atoms in all cases, such as D498 and the backbone oxygen of E497 in BHC80, D212 and D214 in BRPF2, N295 and D297 in AIRE, D88 and D90 in DNMT3L, and E315 and D328 in $\mathrm{DPF} 3 \mathrm{~b}$. In our case, however, this recognition is indirect, resorting to a water molecule for a tetrahedral hydrogen bond network involving two oxygen atoms in E261, one oxygen atom in $\mathrm{N} 274$, and an amino hydrogen in $\mathrm{K} 4$ on H3 (Fig. 2E; Supplemental Fig. S12). The inserted water molecule sheds light on a novel $\mathrm{H} 3 \mathrm{~K} 4 \mathrm{me} 0$ recognition pattern, the introduction of which disrupts the direct polar contacts between PHD finger and $\mathrm{H} 3 \mathrm{~K} 4 \mathrm{me} 0$ therefore allows relatively weak interactions. As a result, unlike previously identified $\mathrm{H} 3 \mathrm{~K} 4 \mathrm{me}$-binding PHD fingers whose recognition would be abrogated totally by the methylation of $\mathrm{H} 3 \mathrm{~K} 4$, the tandem $\mathrm{PHD}$ finger in MOZ tolerates even the trimethylation on H3K4 (Fig. 1C; Supplemental Fig. S3A). This kind of tolerance could also be accounted for by the "induced fit" accommodation within the segment between G272 and N277. Considering the predominant coexistence of $\mathrm{H} 3 \mathrm{~K} 14 \mathrm{ac}$ with methylated $\mathrm{H} 3 \mathrm{~K} 4$ in the promoter region of a transcriptionally active gene, tolerating K4 methylation might indicate a strategy to prevent the active transcription signal inherent in H3K14ac from becoming obscured in the ocean of H3K4 methylation.

\section{Combinatorial action of epigenetic readers in the MOZ complex}

The MOZ complex comprises four components: MOZ, BRPF1/2/3, ING5, and EAF6 (Ullah et al. 2008). Several epigenetic readers are included in the complex, such as the ING5 PHD finger recognizing H3K4me3 (Champagne et al. 2008), BRPF1 PWWP recognizing H3K36me3 (Vezzoli et al. 2010), the BRPF2 PHD finger 1 recognizing $\mathrm{H} 3 \mathrm{~K} 4 \mathrm{me} 0$ (Qin et al. 2011), and the BRPF bromodomain recognizing acetylated histones and other proteins (Zeng and Zhou 2002). Our present work showed that unmodified R2 and acetylated $\mathrm{K} 14$ of the $\mathrm{H} 3$ tail can be specifically read by the tandem PHD finger of MOZ. The presence of multiple epigenetic readers in a single HAT complex raises the following questions: Why are so many histone-binding domains required? How do they cooperate to function? An increasing body of literature indicates that histone modifications are not a simple code, but a sophisticated, nuanced chromatin "language" in which different combinations of basic building blocks yield dynamic functional outcomes through combinatorial reading by epigenetic "readers" (Narlikar et al. 2002; Berger 2007; Ruthenburg et al. 2007b). MOZ acetylates histone $\mathrm{H} 3$ at the K14 position on the HOXA9 promoter (Fig. 6A), while its tandem $\mathrm{PHD}$ finger recognizes the same histone mark (H3K14ac), indicating a positive feedback mechanism underlying the regulation of HOXA9 by MOZ. In detail, after being recruited to the target sites, MOZ uses PHD12 to stabilize the complex localized on the HOXA9 promoter by binding to histone H3. This is supported by the enrichment defect observed in mutant D282A in our ChIP experiments. Then, the MOZ complex acetylates H3 at the K14 position, leading to a tighter binding between PHD12 and H3, which could be supported by a more than twofold affinity increase introduced by acetylation of H3K14 in our in vitro binding assays (Figs. 1C, 3B). H3K14 acetylation further stabilizes the MOZ complex on the target position and facilitates the spread of this modification around. This kind of positive feedback mechanism is also found in histone methyltransferase (HMT) complexes (Ruthenburg et al. 2007a), suggesting a conserved regulatory manner for histone-modifying enzymes.

Generally, high levels of H3K4me3 and histone acetylation such as $\mathrm{H} 3 \mathrm{~K} 14 \mathrm{ac}$ are associated together with the 5 ' regions of transcriptionally active genes. Also, there is a strong positive correlation between these modifications and transcription rates (Ruthenburg et al. 2007a; Wang et al. 2008). Based on recent studies and our current work, we proposed a hypothesis model about combinatorial actions of epigenetic readers in the MOZ complex. Like that in the HBO1 (Saksouk et al. 2009) and NuA3 (Taverna et al. 2006) complex, the PHD finger of ING5 binds histone $\mathrm{H} 3 \mathrm{~K} 4 \mathrm{me} 3$ to recruit the MOZ complex to the target chromatin sites (Fig. 7A; Champagne et al. 2008). After that, the first PHD finger of the BRPF protein binds $\mathrm{H} 3 \mathrm{~K} 4 \mathrm{meO}$ (Qin et al. 2011), and the tandem PHD finger of MOZ binds to unmodified H3R2, stabilizing the complex to perform acetyl transfer reactions against histone H3 (Fig. 7B). Accompanied by chromatin relaxation, the positive feedback mechanism begins to function, spreads the H3K14ac mark around, and finally switches the target gene to its active state (Fig. 7C). Supportive of these findings, another study showed that MOZ and MLL, an H3K4me2/3 HMT complex, interact, colocalize, and functionally cooperate on multiple $H O X$ promoters (Paggetti et al. 2010), highlighting the relationships between MOZ and the modifications of H3K4me3 and H3K14ac.

Notably, methylation of H3R2, like a molecular switch, abrogates the trimethylation of H3K4 by the Set1 methyltransferase (Guccione et al. 2007; Kirmizis et al. 2007) and functions as a negative regulator of $\mathrm{N}$-terminal $\mathrm{H} 3$ tail binding (Iberg et al. 2008). In our case, dimethylation of H3R2 greatly impedes the interactions between the MOZ complex and histone $\mathrm{H} 3$ by abolishing the bindings of PHD fingers of MOZ and ING5 to N-terminal H3 tails. However, more details regarding the H3R2me2-mediated regulation of $\mathrm{MOZ}$ and histone $\mathrm{H} 3$ acetylation depend on future investigations. In summary, unmodified H3R2H3K14ac, perhaps combined with H3K4me3, constitutes a set of histone codes that can be combinatorially recognized by the MOZ complex, and these multivalent interactions 
A

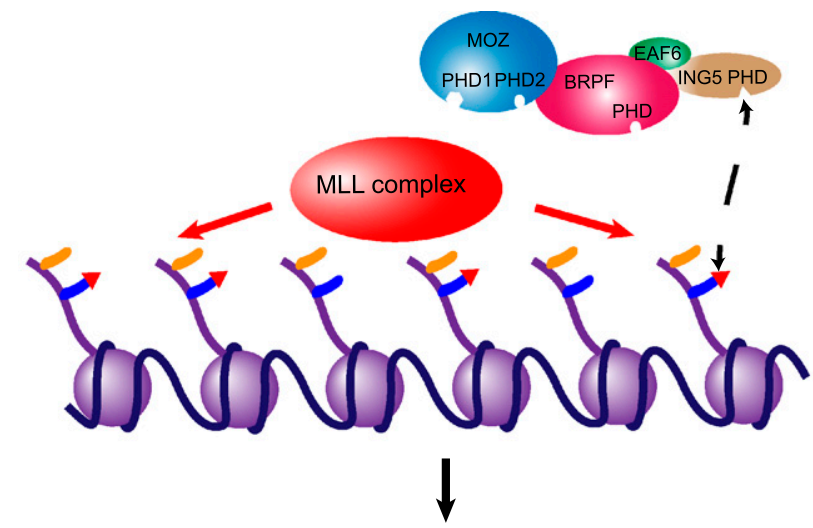

B

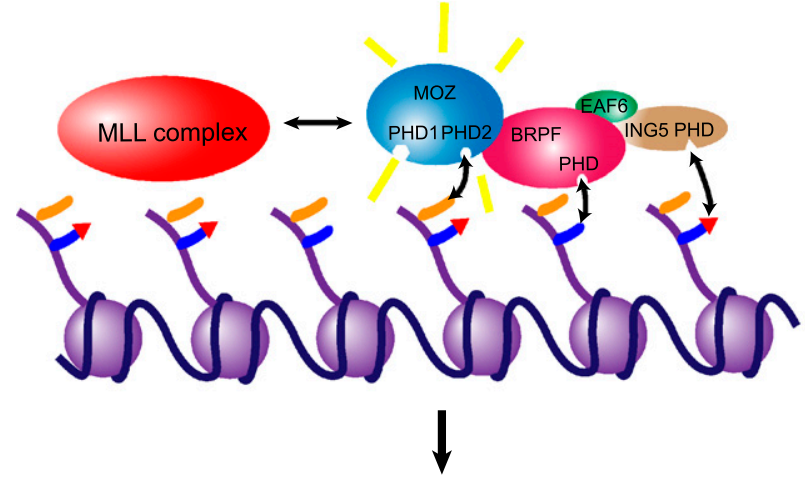

C

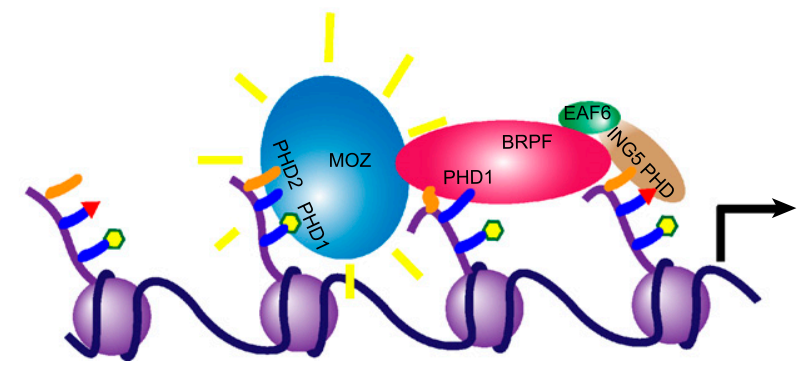

Figure 7. Model for the cross-talk between the MOZ complex and histone modifications. (A) Nucleosomes with the H3K4me3 mark, modified by the MLL complex, recruit the MOZ complex through the binding of the ING5 PHD finger. (B) After that, BRPF PHD1 binds H3K4me0, and MOZ PHD2 binds to unmodified H3R2, stabilizing the complex to perform acetyl transfer reactions against histone H3. (C) Accompanied by chromatin relaxation, the positive feedback mechanism begins to function, spreading the H3K14ac mark around, and finally switches the target gene to its active state.

\footnotetext{
unmodified R2

unmodified K4 or K14

$\checkmark \mathrm{K} 4 \mathrm{me} 3$

$\mathrm{K} 14 \mathrm{ac}$
}

on a single histone tail and beyond may have a significant, if not dominant, role in chromatin transactions.

\section{Materials and methods}

\section{Expression, purification, and isotope labeling of MOZ PHD12}

The DNA fragments of human MOZ tandem PHD finger (residues 204-313) were amplified from the human brain cDNA library (Clontech) by PCR reactions. Then, the DNA fragments were ligated into the NdeI/XhoI-cleaved plasmid pET-28a(+) (Novagen), which was modified with thrombin cleavage site deletion or substitution of a TEV cleavage site, generating fusion proteins with a $6 \mathrm{xHis}$ tag at the $\mathrm{N}$ terminus with or without a TEV cleavage site. MOZ PHD12 mutants were generated by the conventional PCR method using a pET-28a(+)-PHD12 plasmid as template. All of the recombinant MOZ PHD12 proteins were produced in E. coli BL21 (DE3). Cells were grown in either LB medium for unlabeled samples or minimal medium supplemented as appropriate with glucose or $\left[{ }^{13} \mathrm{C}_{6}\right]$ glucose and ${ }^{15} \mathrm{NH}_{4} \mathrm{Cl}$ for ${ }^{15} \mathrm{~N}$ and ${ }^{15} \mathrm{~N} /{ }^{13} \mathrm{C}$ samples, and $0.1 \mathrm{mM} \mathrm{Zn}_{2} \mathrm{SO}_{4}$ was added. Generally, the protein expression was induced at $\mathrm{A} 600=0.8-1.0$ with $0.5 \mathrm{mM}$ IPTG for $24 \mathrm{~h}$ at $16^{\circ} \mathrm{C}$. The expressed recombinant protein was purified using a Ni-chelating column (Qiagen) followed by TEV cleavage (for crystallography only) and Superdex 75 column gel filtration. The purified protein was exchanged to buffer A (20 mM HEPES, $100 \mathrm{mM} \mathrm{NaCl}$ at $\mathrm{pH}$ 8.0) as an NMR sample.

\section{Peptide synthesis}

Peptides H3 (1-10), H3 (1-18), H3 (1-12)R2me2a, H3 (1-12)R2me2s, H3 (1-10)K4me3, H3 (1-15)K9me3, H3 (1-12)K9ac, H3 (1-18)K14, 
H3 (1-18)K14ac, and H4 (11-21)K16ac were used in this study; their sequences are listed in Supplemental Table S3, and they were synthesized by GL Biochem (Shanghai). Biotinylated peptides H3 (1-18), H3 (1-18)R2me2a, H3 (1-18)R2me2s, and H3 (1-18)K14ac were synthesized by Scilight Biotechnology (Beijing). They were supplied with stringent analytical specifications (purity level $>98 \%$ ), which included HPLC and MS analysis. Before use, peptides were weighed and dissolved in buffer $\mathrm{A}$, and the $\mathrm{pH}$ of each sample was systematically controlled and, if necessary, adjusted to a value of 8.0 by addition of diluted $\mathrm{NaOH}$ or a $\mathrm{HCl}$ solution.

\section{NMR spectroscopy}

All spectra were recorded at $25^{\circ} \mathrm{C}$ on a Bruker DMX500 or DMX600 spectrometer. Both the purified ${ }^{15} \mathrm{~N}$-labeled and ${ }^{13} \mathrm{C}$, ${ }^{15} \mathrm{~N}$-labeled proteins were dissolved to a final concentration of $0.8 \mathrm{mM}$ in $500 \mu \mathrm{L}$ of buffer A with $10 \% \mathrm{D}_{2} \mathrm{O}$. All NMR experiments were performed at $293 \mathrm{~K}$ on a Bruker DMX500 or DMX600 spectrometer.

The following spectra were recorded to obtain backbone and side chain resonance assignments (Clore and Gronenborn 1994): two-dimensional (2D) ${ }^{1} \mathrm{H}-{ }^{15} \mathrm{~N}$ HSQC; three-dimensional (3D) triple-resonance spectra $\mathrm{HNCO}, \mathrm{HN}(\mathrm{CA}) \mathrm{CO}, \mathrm{CBCA} / \mathrm{CO} / \mathrm{NH}$, CBCANH, HBHA(CO) NH, C(CO)NH-TOCSY, and $\mathrm{H}(\mathrm{CCO}) \mathrm{NH}-$ TOCSY; 2D ${ }^{1} \mathrm{H}^{13}{ }^{13} \mathrm{CHSQC}$; and 3D HCCH-TOCSY and HCCHCOSY. NOE (nuclear Overhauser effect) distance restraints were generated using ${ }^{15} \mathrm{~N}$-edited NOESY and ${ }^{13} \mathrm{C}$-edited NOESY spectra using $110-\mathrm{msec}$ mixing times. NMR data were processed by NMRPipe and NMRDraw software (Delaglio et al. 1995) and were assigned with Sparky (T.D. Goddard and D.G. Kneller, University of California at San Francisco). The Talos ${ }^{+}$ (Shen et al. 2009) program was used to obtain the backbone dihedral angles $(\Phi$ and $\psi)$ in secondary structures on the basis of chemical shift information. Hydrogen bond restraints were defined from slow-exchanging amide protons identified after exchange of the $\mathrm{H}_{2} \mathrm{O}$ buffer to $\mathrm{D}_{2} \mathrm{O}$.

\section{Structure calculations}

Structure calculation for PHD12 was performed on the basis of proton-proton NOE restraints and dihedral angle $(\Phi$ and $\psi)$ restraints with a simulated annealing protocol using the CNS version 1.2 program (Brunger et al. 1998). High-temperature torsion angle dynamics were performed for 15 psec at 50,000 K (1000 steps), followed by a 15 -psec cooling phase. Initial structure calculations included only hydrogen bonds in defined secondary structures from $\mathrm{Talos}^{+}$. In the following refinement calculation, only hydrogen bonds whose donors could be identified unambiguously were added. In the final calculations, an ensemble of 200 structures (with no distance violations $>0.3 \AA$ and no dihedral angle violations $>5^{\circ}$ ) was generated from unambiguous NOEs previously determined. Twenty models were selected on the basis of energetic criteria (low total energy, using the accept.inp routine) to form a representative ensemble of the calculated structures.

\section{Chemical shift perturbation}

For detecting the histone peptides with several modification binding sites on MOZ PHD12, $0.3 \mathrm{mM}{ }^{15} \mathrm{~N}$-labeled PHD12 was used. After the ${ }^{1} \mathrm{H}^{-15} \mathrm{~N}$ HSQC spectrum of free MOZ PHD12 was recorded, the sample was titrated with histone peptides by the method described by Weber et al. (1991). All HSQC spectra for mapping histone peptide-binding interfaces on MOZ PHD12 were performed on a $600-\mathrm{MHz}$ Bruker at $298 \mathrm{~K}$.
FPAs

FPAs were performed in buffer B (20 mM HEPES, $150 \mathrm{mM} \mathrm{NaCl}$ at $\mathrm{pH} 7.0$ ) at $293 \mathrm{~K}$ using a SpectraMax M5 microplate reader system (Molecular Devices). The wavelengths of fluorescence excitation and emission were $485 \mathrm{~nm}$ and $522 \mathrm{~nm}$, respectively. Each 96-well plate contained $100 \mathrm{nM}$ C-terminal fluorescein isothiocyanate (FITC)-labeled H3K14ac peptide (residues 1-18) probe and different amounts of PHD12 or mutants (concentrations from $0 \mu \mathrm{M}$ to $\sim 100 \mu \mathrm{M}$ ) with a final volume of $200 \mu \mathrm{L}$. For each assay, peptide-free controls (PHD12 or mutants only) were included.

\section{Peptide pull-down assays}

Biotinylated histone peptides ( $1 \mu \mathrm{g}$ each) that were used for the pull-down assays were incubated with $2-5 \mu \mathrm{g}$ of purified GSTPHD12 for $2 \mathrm{~h}$ at room temperature in binding buffer $(20 \mathrm{mM}$ Tris- $\mathrm{HCl}$ at $\mathrm{pH} 7.5,150 \mathrm{mM} \mathrm{NaCl}$ ). After $1 \mathrm{~h}$ of incubation, Streptavidin beads (ThermoScientific) were washed three times using binding buffer with $0.1 \%$ Triton X-100 and were subjected to Coomassie blue staining.

\section{$X$-ray crystallography}

The 6xHis tag of the recombinant MOZ PHD12 protein was removed by TEV enzyme digestion. Then, the protein was purified by Superdex 75 column gel filtration and monoQ anion exchange. The MOZ PHD12 complexes with H3K14ac were prepared by mixing $8.5 \mathrm{mg}$ of PHD12 with $3 \mathrm{mg}$ of $\mathrm{H} 3 \mathrm{~K} 14 \mathrm{ac}(1-18)$ peptide in a $1-\mathrm{mL}$ volume and incubated for a further $15 \mathrm{~min}$ on ice. Crystals were grown at $20^{\circ} \mathrm{C}$ by the sessile droplet vapor diffusion method by mixing $1 \mu \mathrm{L}$ of complex with $1 \mu \mathrm{L}$ of reservoir solution. Crystals for the complex were produced from a reservoir solution containing $0.2 \mathrm{M}$ ammonium acetate, $0.1 \mathrm{M}$ tri-sodium citrate, and $30 \%$ PEG4000 (pH 5.6).

A SAD data set was collected on beam line $17 \mathrm{U}$ at Shanghai Synchrotron Radiation Facilities (SSRF) at a wavelength of $0.97 \AA$, and the data were processed using programs in CCP4 package (Collaborative Computational Project, Number 4 1994). All of the four expected endogenous zinc atom positions were determined by SHELX C/D (Sheldrick 2008), and the initial phases of calculations and density modification were performed with SHELX E (Sheldrick 2008). An initial model was automatically built by the program ARP/wARP (Langer et al. 2008). The model was further built and refined using Coot (Emsley et al. 2010) and Refmac5 (Murshudov et al. 1997), respectively.

\section{HAT activity assay}

The HAT activity of the recombinant PHD-MYST domain and MYST domain were determined using a ${ }^{3} \mathrm{H}$-based radioactive HAT assay as described (Holbert et al. 2007). The substrate utsed in the HAT assay was histone octamer reconstituted as described by Luger et al. (1999). The following reaction protocol was applied for $30-\mu \mathrm{L}$ reaction volumes: Two concentrations of MOZ PHDMYST and its mutants ( 30 or $90 \mathrm{nM}$ ) were incubated with $1 \mu \mathrm{g}$ of histone octamer and $0.25 \mu \mathrm{Ci}$ of Ac-CoA in HAT buffer $(20 \mathrm{mM}$ Tris- $\mathrm{HCl}$ at $\mathrm{pH} 7.6,30 \mathrm{mM}$ sodium chloride, $5 \%$ [v/v] glycerol, $10 \mathrm{mM}$ sodium butyrate, $1 \mathrm{mM}$ phenylmethylsulfonyl fluoride) for $30 \mathrm{~min}$ at $30^{\circ} \mathrm{C}$. Buffer control reactions not including enzyme were also performed. After incubation, the reactions were stopped by adding $5 \times$ SDS sample buffer, separated using SDS-PAGE, and then transferred onto a PVDF membrane. The transferred protein samples were stained by Coomassie brilliant blue R-250, processed by EN3HHANCE spray, and subsequently exposed to 
Amersham HyperfilmTM MP for $24 \mathrm{~h}$ at $-80^{\circ} \mathrm{C}$. The bands on the PVDF membrane corresponding to histone octamer could be subject to scintillation counting after the exposure. For HAT assays of different fragments of $\mathrm{MOZ}$, the concentrations of MOZ fragments and histone octamer were switched to $200 \mathrm{nM}$ and $2 \mu \mathrm{g}$ per reaction, respectively.

\section{Coimmunoprecipitation and Western blot}

Two 6-cm dishes of $293 \mathrm{~T}$ cells (transiently transfected with control Flag plasmid or Flag-PHD12 plasmid) were harvested, washed with PBS, and ultrasonicated in a cold PBS buffer containing protease inhibitor cocktail. After centrifugation, $1 \mathrm{mg}$ of total proteins was then incubated with anti-Flag M2 affinity gel (Sigma) for immunoprecipitating proteins with gentle shaking overnight at $4^{\circ} \mathrm{C}$. Immunoprecipitation complexes were washed twice in icecold PBS buffer. Proteins were eluted by boiling in Laemmli buffer. Eluated proteins were then separated by SDS-PAGE and electroblotted to nitrocellulose membranes. Membranes were blocked in $1 \times$ PBS-T $(0.1 \%)$ and fat-free dry milk or bovine serum albumin (BSA; 5\%) (blocking buffer) for $1 \mathrm{~h}$ at room temperature. Membranes were incubated with the primary antibodies diluted in the blocking buffer overnight at $4^{\circ} \mathrm{C}$. Membranes were washed three times in $1 \times$ PBS-T $(0.1 \%)$ during 10 min each. Secondary antibodies conjugated with horseradish peroxidase were added, and the membranes were incubated for $1 \mathrm{~h}$ at room temperature. Membranes were washed three times in $1 \times$ PBS-T $(0.1 \%)$ for $10 \mathrm{~min}$ each. ECL Western blotting reagent kit (ThermoScientific) was used for protein detection.

\section{Immunostaining and fluorescence colocalization}

Cells were cultured in 24-well chamber slides. After washing once with PBS, cells were fixed in $4 \%$ paraformaldehyde and permeabilized in PBS containing 0.5\% Triton X-100 for 5 min at room temperature. After blocking with 5\% BSA in PBS for $1 \mathrm{~h}$, cells were incubated with primary antibodies and donkey antimouse immunoglobulin $\mathrm{G}$ conjugated with rhodamine antibody (Jackson ImmunoResearch) or goat anti-rabbit immunoglobulin G conjugated with FITC antibody (Jackson ImmunoResearch), respectively. Hoechst 33342 (Sigma) was used for nuclear staining. After extensive washing, cells were analyzed by an Olympus IX71 inverted microscope coupled to an Olympus DP70 high-resolution color camera or by a Zeiss LSM710 laser confocal system.

\section{ChIP}

293 T cells were cultured in DMEM medium supplemented with $10 \%$ fetal bovine serum (Gibco). Transient transfection was performed with X-tremeGENE HP DNA transfection reagent (Roche) according to the manufacturer's instructions. ChIP assays were performed using EZ ChIP kit (Millipore). Briefly, 293T cells $\left(1 \times 10^{7}\right.$ cells $)$ were fixed with $1 \%$ formaldehyde and then neutralized by adding $0.125 \mathrm{M}$ glycine. Cells were collected and lysed in cell lysis buffer containing SDS and cocktail protease inhibitor. The lysate was sonicated to obtain soluble chromatin with an average length of $1000 \mathrm{bp}$. After 1:10 dilution in dilution buffer, chromatin solutions were precleared and then were incubated with IgG, anti-Flag antibody (Sigma), anti-H3 antibody (Abcam), anti-H3K9ac antibody (Millipore), or anti-H3K14ac antibody (Millipore), respectively. Next, the mixture was incubated overnight at $4^{\circ} \mathrm{C}$ on a rotating platform. The immunocomplexes were captured by protein G-Sepharose beads. After extensive washing, the bound DNA fragments were eluted, and the resulting DNA was subjected to real-time qPCR analysis using the primer pairs shown in Supplemental Table S3.

\section{Accession numbers}

The atomic coordinates and structure factors have been deposited in the PDB with the following accession codes: PHD12 in the free state (2LNO) and in complex with H3 (1-18)K14ac peptide (3V43). The Biological Magnetic Resonance Data Bank (BMRB) accession code for the NMR structure of PHD12 is 18142 .

\section{Acknowledgments}

We thank Dr. Xiangiiao Yang and Dr. Xuebiao Yao for offering the wild-type Flag-MOZ plasmid and FITC-labeled anti-rabbit IgG antibody, respectively. We thank Dr. Ronen Marmorstein for giving us the detailed methods for MYST domain purification. We thank Beibei Zhang, Bo Wu, and Haizhen Long for helping with cell culture and nucleosome reconstitution. We also thank the staff at beam line $17 \mathrm{U}$ of Shanghai Synchrotron Radiation Facilities (SSRF) for assistance with X-ray data collection. This work was financially supported by the National Basic Research Program of China (973 Program, grants 2011CB966302, 2012CB917201, and 2011CB911104), the Chinese National Natural Science Foundation (grants 30830031, 31170693, and 91019007 to G.L.), and the Strategic Priority Research Program of the Chinese Academy of Sciences (XDA01010304 to G.L.).

\section{References}

Berger SL. 2007. The complex language of chromatin regulation during transcription. Nature 447: 407-412.

Borrow J, Stanton VP Jr, Andresen JM, Becher R, Behm FG, Chaganti RS, Civin CI, Disteche C, Dube I, Frischauf AM, et al. 1996. The translocation $\mathrm{t}(8 ; 16)(\mathrm{p} 11 ; \mathrm{p} 13)$ of acute myeloid leukaemia fuses a putative acetyltransferase to the CREBbinding protein. Nat Genet 14: 33-41.

Brunger AT, Adams PD, Clore GM, DeLano WL, Gros P, GrosseKunstleve RW, Jiang JS, Kuszewski J, Nilges M, Pannu NS, et al. 1998. Crystallography and NMR system: A new software suite for macromolecular structure determination. Acta Crystallogr 54: 905-921.

Carapeti M, Aguiar RC, Goldman JM, Cross NC. 1998. A novel fusion between $\mathrm{MOZ}$ and the nuclear receptor coactivator TIF2 in acute myeloid leukemia. Blood 91: 3127-3133.

Chaffanet M, Gressin L, Preudhomme C, Soenen-Cornu V, Birnbaum D, Pebusque MJ. 2000. MOZ is fused to p300 in an acute monocytic leukemia with $\mathrm{t}(8 ; 22)$. Genes Chromosomes Cancer 28: 138-144.

Champagne KS, Saksouk N, Pena PV, Johnson K, Ullah M, Yang XJ, Cote J, Kutateladze TG. 2008. The crystal structure of the ING5 PHD finger in complex with an H3K4me3 histone peptide. Proteins 72: 1371-1376.

Clore GM, Gronenborn AM. 1994. Multidimensional heteronuclear nuclear magnetic resonance of proteins. Methods Enzymol 239: 349-363.

Collaborative Computational Project, Number 4. 1994. The Ccp4 suite: Programs for protein crystallography. Acta Crystallogr D Biol Crystallogr 50: 760-763.

Crump JG, Swartz ME, Eberhart JK, Kimmel CB. 2006. Mozdependent Hox expression controls segment-specific fate maps of skeletal precursors in the face. Development 133: 2661-2669.

Delaglio F, Grzesiek S, Vuister GW, Zhu G, Pfeifer J, Bax A. 1995. NMRPipe: A multidimensional spectral processing system based on UNIX pipes. J Biol NMR 6: 277-293.

Dhalluin C, Carlson JE, Zeng L, He C, Aggarwal AK, Zhou MM. 1999. Structure and ligand of a histone acetyltransferase bromodomain. Nature 399: 491-496. 
Downs JA, Nussenzweig MC, Nussenzweig A. 2007. Chromatin dynamics and the preservation of genetic information. Nature 447: 951-958.

Doyon Y, Cayrou C, Ullah M, Landry AJ, Cote V, Selleck W, Lane WS, Tan S, Yang XJ, Cote J. 2006. ING tumor suppressor proteins are critical regulators of chromatin acetylation required for genome expression and perpetuation. Mol Cell 21: 51-64.

Emsley P, Lohkamp B, Scott WG, Cowtan K. 2010. Features and development of Coot. Acta Crystallogr D Biol Crystallogr 66: $486-501$.

Groth A, Rocha W, Verreault A, Almouzni G. 2007. Chromatin challenges during DNA replication and repair. Cell 128: 721733.

Guccione E, Bassi C, Casadio F, Martinato F, Cesaroni M, Schuchlautz H, Luscher B, Amati B. 2007. Methylation of histone H3R2 by PRMT6 and H3K4 by an MLL complex are mutually exclusive. Nature 449: 933-937.

Holbert MA, Sikorski T, Carten J, Snowflack D, Hodawadekar S, Marmorstein R. 2007. The human monocytic leukemia zinc finger histone acetyltransferase domain contains DNA-binding activity implicated in chromatin targeting. I Biol Chem 282: 36603-36613.

Holm L, Rosenstrom P. 2010. Dali server: Conservation mapping in 3D. Nucleic Acids Res 38: W545-W549. doi: 10.1093/ nar/gkq366.

Horton JR, Upadhyay AK, Qi HH, Zhang X, Shi Y, Cheng X. 2010. Enzymatic and structural insights for substrate specificity of a family of jumonji histone lysine demethylases. Nat Struct Mol Biol 17: 38-43.

Iberg AN, Espejo A, Cheng D, Kim D, Michaud-Levesque J, Richard S, Bedford MT. 2008. Arginine methylation of the histone $\mathrm{H} 3$ tail impedes effector binding. I Biol Chem 283: 3006-3010.

Katsumoto T, Aikawa Y, Iwama A, Ueda S, Ichikawa H, Ochiya T, Kitabayashi I. 2006. MOZ is essential for maintenance of hematopoietic stem cells. Genes Dev 20: 1321-1330.

Kirmizis A, Santos-Rosa H, Penkett CJ, Singer MA, Vermeulen M, Mann M, Bahler J, Green RD, Kouzarides T. 2007. Arginine methylation at histone H3R2 controls deposition of H3K4 trimethylation. Nature 449: 928-932.

Kitabayashi I, Aikawa Y, Nguyen LA, Yokoyama A, Ohki M. 2001. Activation of AML1-mediated transcription by MOZ and inhibition by the MOZ-CBP fusion protein. EMBO I 20: 7184-7196.

Kornberg RD, Lorch Y. 1999. Twenty-five years of the nucleosome, fundamental particle of the eukaryote chromosome. Cell 98: 285-294.

Kouzarides T. 2007. Chromatin modifications and their function. Cell 128: 693-705.

Lange M, Kaynak B, Forster UB, Tonjes M, Fischer JJ, Grimm C, Schlesinger J, Just S, Dunkel I, Krueger T, et al. 2008. Regulation of muscle development by DPF3, a novel histone acetylation and methylation reader of the BAF chromatin remodeling complex. Genes Dev 22: 2370-2384.

Langer G, Cohen SX, Lamzin VS, Perrakis A. 2008. Automated macromolecular model building for X-ray crystallography using ARP/wARP version 7. Nat Protoc 3: 1171-1179.

Laue K, Daujat S, Crump JG, Plaster N, Roehl HH, Tubingen Screen C, Kimmel CB, Schneider R, Hammerschmidt $M$. 2008. The multidomain protein Brpf1 binds histones and is required for Hox gene expression and segmental identity. Development 135: 1935-1946.

Liu CL, Kaplan T, Kim M, Buratowski S, Schreiber SL, Friedman N, Rando OJ. 2005. Single-nucleosome mapping of histone modifications in S. cerevisiae. PLoS Biol 3: e328. doi: 10.1371/ journal.pbio.0030328.
Luger K, Rechsteiner TJ, Richmond TJ. 1999. Preparation of nucleosome core particle from recombinant histones. Methods Enzymol 304: 3-19.

Mansfield RE, Musselman CA, Kwan AH, Oliver SS, Garske AL, Davrazou F, Denu JM, Kutateladze TG, Mackay JP. 2011. Plant homeodomain (PHD) fingers of CHD4 are histone H3binding modules with preference for unmodified H3K4 and methylated H3K9. J Biol Chem 286: 11779-11791.

Martin DG, Grimes DE, Baetz K, Howe L. 2006. Methylation of histone $\mathrm{H} 3$ mediates the association of the NuA3 histone acetyltransferase with chromatin. Mol Cell Biol 26: 30183028.

Miller CT, Maves L, Kimmel CB. 2004. moz regulates Hox expression and pharyngeal segmental identity in zebrafish. Development 131: 2443-2461.

Mishima Y, Miyagi S, Saraya A, Negishi M, Endoh M, Endo TA, Toyoda T, Shinga J, Katsumoto T, Chiba T, et al. 2011. The Hbo1-Brd1/Brpf2 complex is responsible for global acetylation of H3K14 and required for fetal liver erythropoiesis. Blood 118: 2443-2453.

Murshudov GN, Vagin AA, Dodson EJ. 1997. Refinement of macromolecular structures by the maximum-likelihood method. Acta Crystallogr D Biol Crystallogr 53: 240-255.

Narlikar GJ, Fan HY, Kingston RE. 2002. Cooperation between complexes that regulate chromatin structure and transcription. Cell 108: 475-487.

Owen DI, Ornaghi P, Yang JC, Lowe N, Evans PR, Ballario P, Neuhaus D, Filetici P, Travers AA. 2000. The structural basis for the recognition of acetylated histone $\mathrm{H} 4$ by the bromodomain of histone acetyltransferase gen5p. EMBO J 19: 6141-6149.

Paggetti J, Largeot A, Aucagne R, Jacquel A, Lagrange B, Yang XJ, Solary E, Bastie JN, Delva L. 2010. Crosstalk between leukemia-associated proteins MOZ and MLL regulates HOX gene expression in human cord blood CD34+ cells. Oncogene 29: 5019-5031.

Palacios A, Garcia P, Padro D, Lopez-Hernandez E, Martin I, Blanco FJ. 2006. Solution structure and NMR characterization of the binding to methylated histone tails of the plant homeodomain finger of the tumour suppressor ING4. FEBS Lett 580: 6903-6908.

Pelletier N, Champagne N, Stifani S, Yang XJ. 2002. MOZ and MORF histone acetyltransferases interact with the Runtdomain transcription factor Runx2. Oncogene 21: 27292740.

Pena PV, Davrazou F, Shi X, Walter KL, Verkhusha VV, Gozani O, Zhao R, Kutateladze TG. 2006. Molecular mechanism of histone H3K4me3 recognition by plant homeodomain of ING2. Nature 442: 100-103.

Pokholok DK, Harbison CT, Levine S, Cole M, Hannett NM, Lee TI, Bell GW, Walker K, Rolfe PA, Herbolsheimer E, et al. 2005. Genome-wide map of nucleosome acetylation and methylation in yeast. Cell 122: 517-527.

Qin S, Jin L, Zhang J, Liu L, Ji P, Wu M, Wu J, Shi Y. 2011. Recognition of unmodified histone $\mathrm{H} 3$ by the first $\mathrm{PHD}$ finger of bromodomain-PHD finger protein 2 provides insights into the regulation of histone acetyltransferases monocytic leukemic zinc-finger protein (MOZ) and MOZ-related factor (MORF). J Biol Chem 286: 36944-36955.

Rosaleny LE, Ruiz-Garcia AB, Garcia-Martinez J, Perez-Ortin JE, Tordera V. 2007. The Sas3p and Gcn5p histone acetyltransferases are recruited to similar genes. Genome Biol 8: R119. doi: 10.1186/gb-2007-8-6-r119.

Ruthenburg AJ, Allis CD, Wysocka J. 2007a. Methylation of lysine 4 on histone H3: Intricacy of writing and reading a single epigenetic mark. Mol Cell 25: 15-30. 
Ruthenburg AJ, Li H, Patel DJ, Allis CD. 2007b. Multivalent engagement of chromatin modifications by linked binding modules. Natl Rev 8: 983-994.

Saksouk N, Avvakumov N, Champagne KS, Hung T, Doyon Y, Cayrou C, Paquet E, Ullah M, Landry AJ, Cote V, et al. 2009. HBO1 HAT complexes target chromatin throughout gene coding regions via multiple PHD finger interactions with histone H3 tail. Mol Cell 33: 257-265.

Sheldrick GM. 2008. A short history of SHELX. Acta Crystallogr A 64: 112-122.

Shen Y, Delaglio F, Cornilescu G, Bax A. 2009. TALOS ${ }^{+}$: A hybrid method for predicting protein backbone torsion angles from NMR chemical shifts. J Biol NMR 44: 213-223.

Shi X, Hong T, Walter KL, Ewalt M, Michishita E, Hung T, Carney D, Pena P, Lan F, Kaadige MR, et al. 2006. ING2 PHD domain links histone $\mathrm{H} 3$ lysine 4 methylation to active gene repression. Nature 442: 96-99.

Taverna SD, Ilin S, Rogers RS, Tanny JC, Lavender H, Li H, Baker L, Boyle I, Blair LP, Chait BT, et al. 2006. Yng1 PHD finger binding to $\mathrm{H} 3$ trimethylated at $\mathrm{K} 4$ promotes $\mathrm{NuA} 3$ HAT activity at $\mathrm{K} 14$ of $\mathrm{H} 3$ and transcription at a subset of targeted ORFs. Mol Cell 24: 785-796.

Thomas T, Corcoran LM, Gugasyan R, Dixon MP, Brodnicki T, Nutt SL, Metcalf D, Voss AK. 2006. Monocytic leukemia zinc finger protein is essential for the development of longterm reconstituting hematopoietic stem cells. Genes Dev 20: $1175-1186$

Ullah M, Pelletier N, Xiao L, Zhao SP, Wang K, Degerny C, Tahmasebi S, Cayrou C, Doyon Y, Goh SL, et al. 2008. Molecular architecture of quartet MOZ/MORF histone acetyltransferase complexes. Mol Cell Biol 28: 6828-6843.

Vezzoli A, Bonadies N, Allen MD, Freund SM, Santiveri CM, Kvinlaug BT, Huntly BJ, Gottgens B, Bycroft M. 2010. Molecular basis of histone H3K36me3 recognition by the PWWP domain of Brpf1. Nat Struct Mol Biol 17: 617-619.

Voss AK, Collin C, Dixon MP, Thomas T. 2009. Moz and retinoic acid coordinately regulate H3K9 acetylation, Hox gene expression, and segment identity. Dev Cell 17: 674-686.

Wang GG, Allis CD, Chi P. 2007. Chromatin remodeling and cancer, part I: Covalent histone modifications. Trends Mol Med 13: 363-372.

Wang Z, Zang C, Rosenfeld JA, Schones DE, Barski A, Cuddapah S, Cui K, Roh TY, Peng W, Zhang MQ, et al. 2008. Combinatorial patterns of histone acetylations and methylations in the human genome. Nat Genet 40: 897-903.

Weber C, Wider G, von Freyberg B, Traber R, Braun W, Widmer H, Wuthrich K. 1991. The NMR structure of cyclosporin A bound to cyclophilin in aqueous solution. Biochemistry 30: 6563-6574.

Yang XJ, Ullah M. 2007. MOZ and MORF, two large MYSTic HATs in normal and cancer stem cells. Oncogene 26: 54085419.

Zeng L, Zhou MM. 2002. Bromodomain: An acetyl-lysine binding domain. FEBS Lett 513: 124-128.

Zeng L, Zhang Q, Li S, Plotnikov AN, Walsh MJ, Zhou MM. 2010. Mechanism and regulation of acetylated histone binding by the tandem PHD finger of DPF3b. Nature 466: 258-262. 


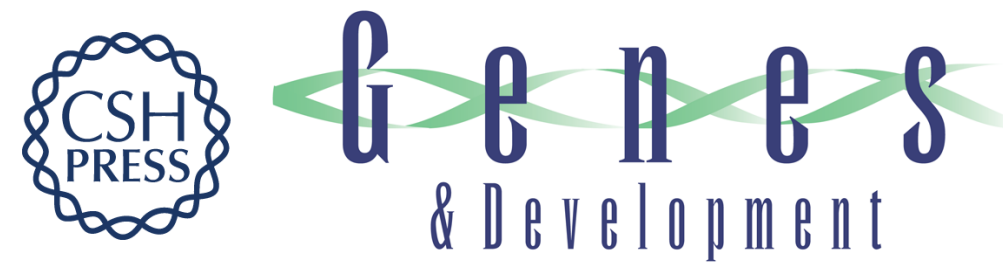

\section{Combinatorial readout of unmodified $\mathrm{H} 3 \mathrm{R} 2$ and acetylated $\mathrm{H} 3 \mathrm{~K} 14$ by the tandem PHD finger of MOZ reveals a regulatory mechanism for HOXA9 transcription}

Yu Qiu, Lei Liu, Chen Zhao, et al.

Genes Dev. 2012, 26:

Access the most recent version at doi:10.1101/gad.188359.112

Supplemental http://genesdev.cshlp.org/content/suppl/2012/06/19/26.12.1376.DC1

Material

References This article cites 61 articles, 16 of which can be accessed free at:

http://genesdev.cshlp.org/content/26/12/1376.full.html\#ref-list-1

License

Email Alerting Receive free email alerts when new articles cite this article - sign up in the box at the top

Service right corner of the article or click here.

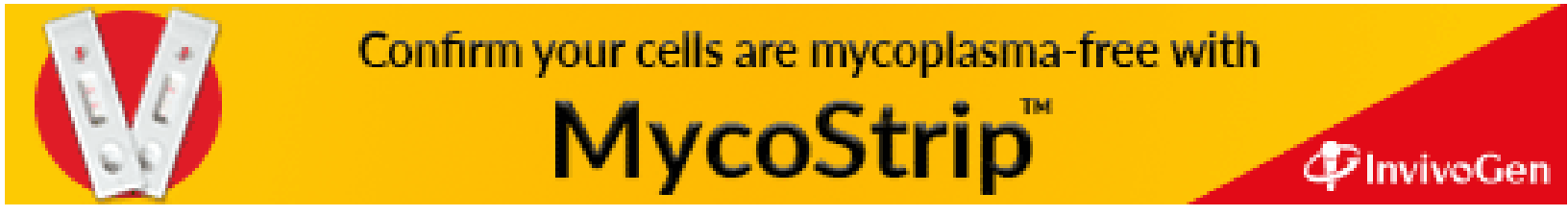

